\title{
CONOCIMIENTOS ASTRONÓMICOS DEL PROFESORADO DE EDUCACIÓN SECUNDARIA OBLIGATORIA Y PREFERENCIAS METODOLÓGICAS PARA LA ENSENANZA DE ASTRONOMÍA
}

\author{
Compulsory secondary education teachers astronomical \\ knowledge and methodological preferences for astronomy \\ teaching
}

Connaissances astronomiques des professeurs d'enseignement secondaire obligatoire et préférences méthodologiques de l'enseignement de l'astronomie

José Luis GARCía HerRero

Profesor de Matemáticas del IES Carmen Martín Gaite (Navalcarnero). Doctorando de la Facultad de Educación de la UNED. Correo-e: jlgarciaberrero@yahoo.es

Recibido: 01-08-2013; Aceptado: 27-10-2013; Publicado: 30-03-2014

BIBLID [0212-5374 (2014) 32, 1; 161-198]

Ref. Bibl. JOSÉ LUIS GARCÍA HERRERO. Conocimientos astronómicos del profesorado de Educación Secundaria Obligatoria y preferencias metodológicas para la enseñanza de astronomía. Enseñanza \& Teaching, 32, 1-2014, 161-198.

RESUMEN: La astronomía forma parte del currículo de las materias científicas en la educación obligatoria, incluyendo básicamente unos pocos conceptos tradicionales como las estaciones del año, las fases lunares, los movimientos de la Tierra, los eclipses..., y dejando de lado otros más actuales como los exoplanetas y la cosmología. 

Y PREFERENCIAS METODOLÓGICAS PARA LA ENSEÑANZA DE ASTRONOMÍA

Este artículo pretende estudiar en primer lugar el nivel de conocimientos básicos sobre astronomía del profesorado de Educación Secundaria Obligatoria (ESO), con el fin de detectar posibles carencias formativas y concepciones alternativas. En el estudio participaron profesores de especialidades científicas y no científicas de dicha etapa. Posteriormente se realizó un análisis de correlación de resultados en función de diversas variables, y además se comparó con los resultados obtenidos por un extenso grupo de alumnos de Eso.

En segundo lugar, el artículo se centra en estudiar las preferencias metodológicas para la enseñanza-aprendizaje de astronomía, contando con los mismos participantes que en el caso anterior, y con el objetivo de conocer sus valoraciones sobre diferentes propuestas, en algunos casos bastante innovadoras.

Los resultados obtenidos pueden ser orientativos para estudios posteriores de mayor envergadura, de cara a la determinación de necesidades de formación específicas en astronomía en el caso de docentes de materias científicas. Además, serán de utilidad como orientaciones metodológicas a la hora de abordar la enseñanza de la astronomía.

Palabras clave: astronomía, conocimientos, metodología de enseñanza-aprendizaje, profesores, alumnos, educación obligatoria.

SUMMARY: Astronomy is part of the scientific curriculum in compulsory education. It includes some basic traditional concepts as seasons, lunar phases, the movements of the Earth, eclipses..., but leaves aside some other more contemporary concepts such as exoplanets and cosmology.

In the first place, this paper focuses on the level of basic knowledge on astronomy basic concepts by secondary education (ESO) teachers, in order to detect possible training gaps and alternative conceptions. The study included teachers of scientific and non-scientific specialities from ESO. Afterwards a correlation analysis was carried out based on several variables, including a comparison with the results obtained by a large group of ESO students.

Secondly, the article focuses on studying the methodological preferences for teaching and learning Astronomy, with the same participants as in the previous case, aiming to know their ratings on various proposals, some of them quite innovative.

The results can be of use as guidance for further studies of larger samples, in order to determine specific training needs in Astronomy in the case of teachers of science subjects. In addition, they will also be useful as methodological guidance in addressing the teaching of astronomy.

Key words: Astronomy, knowledge, teaching-learning methodology, teachers, students, compulsory education.

RÉSUMÉ: L'astronomie fait partie du curriculum scientifique dans l'enseignement obligatoire, et comprend quelques concepts traditionnels comme les saisons, les phases lunaires, les mouvements de la terre, les éclipses..., en laissant de côté d'autres plus actuels comme les exoplanètes et la cosmologie. 
CONOCIMIENTOS ASTRONÓMICOS DEL PROFESORADO DE EDUCACIÓN SECUNDARIA OBLIGATORIA Y PREFERENCIAS METODOLÓGICAS PARA LA ENSEÑANZA DE ASTRONOMÍA

Cet article étudie d'abord le niveau de connaissances de base sur l'astronomie des enseignants d'enseignement secondaire obligatoire (ESO), afin de détecter des lacunes en matière de formation et des conceptions fausses. L'étude a inclus les enseignants des disciplines scientifiques et non scientifiques de ESO. Après cela, une étude de corrélation des résultats a été faite, basé sur plusieurs variables, et aussi par rapport aux résultats obtenus par un grand groupe d'étudiants de ESO.

Deuxièmement, l'article se concentre sur l'étude des préférences méthodologiques pour l'enseignement et l'apprentissage de l'astronomie, avec les mêmes participants que dans le cas précédent, et dans le but de connaître leurs avis sur les différentes propositions, dans certains cas tout à fait novatrices.

Les résultats peuvent être utiles comme des orientations pour des études des échantillonnages plus grands, afin de déterminer les besoins spécifiques de formation en astronomie dans le cas des enseignants des matières scientifiques. Ils seront également utiles comme des conseils méthodologiques pour traiter l'enseignement de l'astronomie.

Mots clés: Astronomie, connaissance, méthodes d'enseignement apprentissage, professeurs, étudiants, instruction obligatoire.

\section{LA ASTRONOMÍA EN LA ESCUELA}

La astronomía es una de las ciencias más antiguas, pues desde tiempos inmemorables la humanidad ha alzado la vista al cielo para preguntarse por su funcionamiento y su sentido. Los interrogantes relacionados con la astronomía han sido uno de los motores fundamentales del desarrollo de la ciencia, y fueron imprescindibles para el inicio de la revolución científica que se fraguó hace cuatro siglos con Copérnico y Galileo como referentes principales.

En la actualidad, nuestra sociedad ha perdido el hábito de observar el cielo, en gran parte debido a la concentración de la población en zonas urbanas con altos índices de contaminación lumínica. Además, la astronomía suscita a menudo una especie de miedo reverencial por la aparente inaccesibilidad de sus conceptos y por la enormidad del Universo. Sin embargo, ninguna ciencia es tan accesible para los aficionados y tan fértil para la divulgación, pues está indisolublemente ligada a nuestra vida en aspectos como el día y la noche, las estaciones del año, los calendarios, las mareas... Sin olvidar el enorme interés que suscitan el origen y la evolución del Universo.

La enseñanza de la astronomía, en sus múltiples vertientes, puede ser una herramienta de gran utilidad para la consecución de las competencias básicas introducidas en la Ley Orgánica de Educación (LOE, 2006) para Educación Primaria (EP) y Educación Secundaria Obligatoria (ESO). El proceso de enseñanza de la astronomía se puede abordar desde diversos puntos de vista y perspectivas metodológicas en función de los objetivos establecidos y de los recursos didácticos disponibles. En España, Arribas y Granados (1992) propusieron una serie de temas en torno a los cuales llevar a la práctica la asignatura optativa Taller de Astronomía, 

Y PREFERENCIAS METODOLÓGICAS PARA LA ENSEÑANZA DE ASTRONOMÍA

como la historia de la astronomía, la construcción de maquetas, la astrofísica, el sistema solar, la mitología celeste, observaciones nocturnas... En la práctica, la metodología más comúnmente utilizada por el profesorado español es la lección magistral con el apoyo del libro de texto (Pérez, Álvarez y Prieto, 2008). Sin embargo, existen estudios que ponen de manifiesto la importancia de llevar a la práctica actividades como las visitas a planetarios (Dunlop, 2000), y analizan diversas estrategias metodológicas como las observaciones directas del cielo (Foster, 1996; Brandou, 1997), el uso de simuladores informáticos (Kanssagalo, 1994), la ilustración de las fases lunares por medio de vídeos (Marshall, 1996) o el potencial de llevar a cabo proyectos de investigación con telescopios profesionales para fomentar el interés por la ciencia (Beare, 2007).

Con respecto a qué contenidos sobre astronomía se deberían enseñar, existe cierta controversia sobre si conviene ceñirse a los tradicionales, como la comprensión de las fases lunares y las estaciones del año, o tratar contenidos relacionados con los temas más de actualidad en astrofísica y cosmología, como los exoplanetas, la evolución estelar, los agujeros negros, el Big Bang, la expansión del Universo... Los defensores de la primera opción argumentan que tras años de formación, los estudiantes no llegan a comprender los fenómenos astronómicos más sencillos, por lo que no merece la pena presentarles conceptos más complejos que tampoco entenderán (Sadler, 2001). Sin embargo, los defensores del segundo enfoque argumentan que se debe mostrar a los estudiantes la comprensión actual del Universo tal y como se conoce en la actualidad (Pasachoff, 2002).

En cualquier caso, si nos ceñimos a EP y ESO en la Comunidad de Madrid, encontraremos que los conceptos relacionados con la astronomía se encuentran muy encorsetados en el currículo oficial (Comunidad de Madrid, 2007a, 2007b) y adoptan un enfoque de contenidos tradicionales.

En el caso de EP, la astronomía se delimita dentro del área Conocimiento del medio natural, social y cultural:

- Primer Ciclo

- Los astros, las estrellas, el Sol, la Tierra y la Luna. La sucesión del día y la noche.

- El tiempo atmosférico. La sucesión estacional.

- Nociones básicas de orientación espacial.

- Las nociones básicas de tiempo y su medida.

- Uso del calendario para señalar hechos de la vida cotidiana.

- Segundo Ciclo

- El planeta Tierra. Características físicas. Océanos, mares y continentes. La representación de la Tierra: el globo terráqueo, mapas e imágenes. Los movimientos de la Tierra y fases de la Luna. Las estaciones del año. 
CONOCIMIENTOS ASTRONÓMICOS DEL PROFESORADO DE EDUCACIÓN SECUNDARIA OBLIGATORIA Y PREFERENCIAS METODOLÓGICAS PARA LA ENSEÑANZA DE ASTRONOMÍA

- Orientación en el espacio. Los puntos cardinales y la brújula. Uso de croquis, planos y mapas.

- Comportamiento de los cuerpos ante la luz. Reflexión y refracción. Descomposición de la luz blanca. El color. Identificación de los colores básicos.

- $\quad$ Tercer Ciclo

- El universo. El sistema solar. Las capas de la Tierra: hidrosfera, corteza, manto y núcleo.

En ESO, la astronomía queda relegada a unas cuantas nociones básicas bastante repetitivas con respecto a los contenidos de EP, como parte de las asignaturas Ciencias de la Naturaleza y Ciencias Sociales de 1.ํㅡㅇ. Aunque existe la posibilidad de introducir la asignatura optativa Taller de Astronomía en 3. ESO, son muy pocos los centros que la ofertan, muy probablemente por la escasez de profesorado suficientemente preparado para su impartición. Aparecen también contenidos de astronomía en la asignatura Física y Química, pero al formar parte de un itinerario específico de $4 .^{\circ} \mathrm{ESO}$, no todos los alumnos de ESO tienen la oportunidad de estudiarlos.

- Ciencias Naturales, 1.ํㅡ ESO:

- La observación del Universo: planetas, estrellas y galaxias.

- La Vía Láctea y el Sistema Solar.

- Características físicas de la Tierra y de los otros componentes del Sistema Solar.

- Los movimientos de la Tierra: las estaciones, el día y la noche, los eclipses y las fases de la Luna.

- Utilización de técnicas de orientación. Observación del cielo diurno y nocturno.

- Evolución histórica de las concepciones sobre el lugar de la Tierra en el Universo: el paso del geocentrismo al heliocentrismo como primera y gran revolución científica.

- Ciencias Sociales, Geografía e Historia, 1.ำ ESO:

- La Tierra, un planeta del Sistema Solar. Los movimientos de la Tierra y sus consecuencias geográficas.

- Física y Química, 4.ํㅡㄹ은

Bloque 2. Fuerzas y movimiento.

- La ley de la Gravitación Universal y la culminación de la primera de las revoluciones científicas. El peso de los cuerpos y su caída. El movimiento de planetas y satélites. 


\section{OBJETIVOS}

Las ideas científicas preconcebidas han recibido diferentes denominaciones por parte de los investigadores en la materia, aunque la más ampliamente aceptada es concepciones alternativas (Abimbola, 1988; Gilbert y Swift, 1985). La mayor parte de la ciudadanía alberga concepciones alternativas sobre fenómenos astronómicos muy comunes, y el profesorado parece no ser una excepción, si atendemos a diversos estudios sobre las concepciones alternativas de estudiantes y profesores de diferentes países sobre fenómenos como el ciclo día-noche, las fases lunares o las estaciones del año. Entre ellos, podemos mencionar como base de la presente investigación los llevados a cabo por Barnett y Morran (2002), Baxter (1989), Danaia y McKinnon (2008), Jones (1988), Jones, Lynch y Reesink (1987), Schneps (1989), Trumper (2000, 2001) y Vega (2001, 2007).

Por otra parte, existen diferentes propuestas de estrategias o metodologías validadas para conseguir una enseñanza-aprendizaje de calidad. Según Sevillano (1998, 2008) podemos mencionar, entre otras, las estrategias constructivistas, de motivación, cooperación, socialización, trabajo autónomo, creatividad, juego, investigación y desarrollo de valores y actitudes. La elección de la estrategia adecuada en cada caso será determinante para el éxito del proceso de enseñanzaaprendizaje, y en el caso de la astronomía se puede hacer uso de un amplio abanico de posibilidades.

Teniendo en cuenta estos factores, el presente trabajo de investigación pretende abordar los siguientes objetivos, en todo caso referidos al ámbito de la Comunidad de Madrid:

- Estudiar los conocimientos sobre conceptos básicos de astronomía del profesorado de ESO.

- Analizar las preferencias metodológicas para la enseñanza-aprendizaje de la astronomía del profesorado de ESO y del alumnado de 3.ํ y $4 . .^{\circ}$ ESO.

\section{Metodología}

En vista de los objetivos planteados, se considera la población docente compuesta por el profesorado de ESO, y la población estudiantil formada por los alumnos de los dos últimos cursos de ESO. Los datos publicados al respecto por la Comunidad de Madrid (2013) permiten realizar una estimación de aproximadamente 28.000 profesores. En el caso de la población estudiantil, la cifra se sitúa en torno a 110.000 alumnos.

De igual manera, teniendo en cuenta que los objetivos de la investigación hacían deseable poder contar con una muestra del mayor tamaño posible, y tras descartar diversos métodos de investigación en educación como entrevistas y grupos de discusión, ya que sólo se habría podido contar con un número reducido de docentes, se optó por elaborar un cuestionario en línea sobre conocimientos de astronomía y preferencias metodológicas. 
CONOCIMIENTOS ASTRONÓMICOS DEL PROFESORADO DE EDUCACIÓN SECUNDARIA OBLIGATORIA Y PREFERENCIAS METODOLÓGICAS PARA LA ENSEÑANZA DE ASTRONOMÍA

En base a estas premisas, para llevar a cabo el estudio sobre conocimientos de conceptos astronómicos del profesorado se diseñó un cuestionario de 22 respuestas cerradas, por medio de la adaptación de una prueba ampliamente reconocida, el Astronomy Diagnostic Test (Hufnagel, 2002; Zeilik, 2003; Bailey y Slater, 2004), así como de las preguntas introductorias a los temas del curso en línea Observaciones y modelos en astronomía (Martínez, Pérez y Boix, 2003), galardonado con el segundo premio del Instituto Nacional de Tecnologías Educativas y de Formación del Profesorado (INTEF) a Materiales Educativos. En el caso del estudio de las preferencias metodológicas en el proceso de enseñanza-aprendizaje de la astronomía, se utilizó un cuestionario de escala Likert, con el objetivo de estudiar el grado de idoneidad de las diferentes metodologías propuestas.

Como parte de una investigación más amplia, los alumnos también respondieron un cuestionario de conceptos astronómicos que incluía las preguntas respondidas por el profesorado, lo cual permitió comparar las puntuaciones obtenidas por profesores y alumnos. En este caso, el cuestionario no se alojó en Internet, sino que los alumnos lo completaron en clase gracias a la inestimable colaboración de varios compañeros docentes.

La adaptación de los dos instrumentos de partida para la confección final del cuestionario se realizó a partir de una prueba piloto con un grupo de 30 alumnos y 5 profesores. Se llevaron a cabo mediciones de los índices de dificultad y discriminación (Morales, 2010) de las preguntas del cuestionario, y se estudió qué parte del cuestionario sobre conceptos astronómicos convenía hacer coincidir en el caso de profesores y alumnos, con el fin de poder elaborar análisis comparativos. Adicionalmente, se contó con la valiosa colaboración de dos profesores expertos en la enseñanza y divulgación de la astronomía, que revisaron el cuestionario inicial y realizaron algunas recomendaciones relacionadas con la dificultad asociada a ciertas preguntas sobre astronomía y con la introducción de alguna propuesta metodológica adicional.

El tamaño de las muestras disponibles fue de 50 profesores y 276 alumnos. Teniendo en cuenta las poblaciones y fijado el nivel de confianza en el 95\%, el error de estimación asociado a estas muestras se sitúa en el 14\% en el caso del profesorado, y en el 6\% para el alumnado.

La muestra de 50 profesores estaba compuesta por docentes de centros públicos y concertados, caracterizada del siguiente modo:

\begin{tabular}{|c|c|c|c|}
\hline \multicolumn{2}{|c|}{ DISTRIBUCIÓN DEL PROFESORADO POR ESPECIALIDAD Y GÉNERO } \\
\hline \multicolumn{2}{|c|}{ Ciencias } & \multicolumn{2}{c|}{ No Ciencias } \\
\hline \multicolumn{2}{|c|}{40} & Varones & Mujeres \\
\hline Varones & 17 & 3 & 7 \\
\hline 23 & Mujeres & \\
\hline
\end{tabular}


CONOCIMIENTOS ASTRONÓMICOS DEL PROFESORADO DE EDUCACIÓN SECUNDARIA OBLIGATORIA Y PREFERENCIAS METODOLÓGICAS PARA LA ENSEÑANZA DE ASTRONOMÍA

La muestra de 276 alumnos estaba compuesta por alumnos de $3 . .^{\circ}$ y $4 .^{\circ}$ ESO procedentes de tres institutos públicos de Getafe, Madrid (sección bilingüe en lengua inglesa) y Navalcarnero, de acuerdo a la siguiente distribución:

\begin{tabular}{|c|c|c|c|c|c|}
\hline \multicolumn{5}{|c|}{ DISTRIBUCIÓN DEL ALUMNADO POR LOCALIDAD/BILINGÜISMO/GÉNERO } \\
\hline Madrid (sección bilingüe) & \multicolumn{2}{|c|}{ Getafe } & \multicolumn{2}{c|}{ Navalcarnero } \\
\hline \multicolumn{2}{|c|}{44} & \multicolumn{2}{|c|}{97} & \multicolumn{2}{c|}{135} \\
\hline VARONES & MUJERES & VARONES & MUJERES & VARONES & MUJERES \\
\hline 23 & 21 & 54 & 43 & 62 & 73 \\
\hline
\end{tabular}

La fiabilidad de los cuestionarios se analizó por medio del cálculo del alfa de Cronbach, siendo el umbral de aceptación del índice de fiabilidad variable en función de los autores. En general, son aceptables índices de fiabilidad a partir de 0,70 (Kline, 1999), situándose los valores comprendidos entre 0,60 y 0,70 en el límite de aceptación (Kerlinger, 1979; Thorndike, 1997; Hair et al., 2006).

A continuación se muestran los valores obtenidos en la presente investigación, pudiéndose constatar que se sitúan dentro de los márgenes de fiabilidad. Los resultados de los análisis completos de la fiabilidad de los cuestionarios se incluyen como Anexo III al final del artículo.

\begin{tabular}{|c|l|c|}
\hline \multicolumn{2}{|c|}{ ÍnDICES DE FIABILIDAD DE LOS CUESTIONARIOS } & ALFA DE CRONBACH \\
\hline \multirow{2}{*}{ Profesorado } & Conceptos astronómicos & 0,77 \\
\cline { 2 - 3 } & Preferencias metodológicas & 0,75 \\
\hline \multirow{2}{*}{ Alumnado } & Conceptos astronómicos & 0,61 \\
\cline { 2 - 3 } & Preferencias metodológicas & 0,74 \\
\hline
\end{tabular}

El cuestionario también incluyó preguntas relacionadas con el uso de las TIC, como parte de una investigación más amplia, pero su análisis queda fuera del objeto del presente artículo. El cuestionario completo del profesorado, que fue alojado en Internet para poderlo hacer accesible al mayor número de docentes posible, se incluye como Anexo I al final del artículo. No se considera necesario incluir el cuestionario del alumnado porque todas sus preguntas objeto de análisis en esta investigación se encuentran contenidas en el cuestionario del profesorado.

El tratamiento estadístico de los datos se realizó por medio de los programas SPSS 17.0 y Excel 2002. En primer lugar, con relación al cuestionario sobre conceptos astronómicos, se llevó a cabo un análisis de frecuencias relativas con su correspondiente representación gráfica, con el fin de visualizar de manera clara 
CONOCIMIENTOS ASTRONÓMICOS DEL PROFESORADO DE EDUCACIÓN SECUNDARIA OBLIGATORIA Y PREFERENCIAS METODOLÓGICAS PARA LA ENSEÑANZA DE ASTRONOMÍA

los porcentajes de acierto y detectar posibles concepciones alternativas. Además, se calculó el coeficiente de correlación biserial-puntual $\left(\mathrm{r}_{\mathrm{bp}}\right)$ para determinar la relación entre diversas variables, analizando los porcentajes globales de acierto en función de las mismas. En segundo lugar, para el estudio de las preferencias metodológicas también se realizó un análisis de frecuencias, complementado con el contraste de medias por medio de análisis de varianza en función de diversas variables, tanto del profesorado como del alumnado.

\section{RESUlTADOS}

En primer lugar, informaremos de los resultados obtenidos por el profesorado en todas las preguntas del cuestionario, desglosando las puntuaciones en función de la especialidad, científica o no, de los docentes participantes en el estudio. En el Anexo II, al final del artículo, se muestran los resultados globales detallados. Además, se analizará el grado de relación existente entre la puntuación obtenida y diversas variables dicotómicas.

En segundo lugar, se mostrarán los resultados obtenidos con relación a las preferencias metodológicas de profesores y alumnos, tanto a nivel descriptivo como por medio del análisis de varianza.

\subsection{Conceptos astronómicos}

Comenzaremos por informar sobre las puntuaciones medias (normalizadas sobre 10 puntos) obtenidas por el profesorado en el cuestionario sobre conocimientos de astronomía, que se muestran en la siguiente tabla:

\begin{tabular}{|l|c|}
\hline \multicolumn{1}{|c|}{ TIPO DE PROFESORADO } & NOTA SOBRE 10 \\
\hline Ciencias & 7,0 \\
\hline No Ciencias & 4,8 \\
\hline Varones & 7,2 \\
\hline Mujeres & 5,7 \\
\hline Ciencias Varones & 7,4 \\
\hline Ciencias Mujeres & 6,3 \\
\hline
\end{tabular}

Como es lógico, se aprecia que la puntuación media del profesorado de Ciencias es sensiblemente superior al resto, aunque cabría esperar mejores resultados. También se observa que en la comparativa entre sexos es superior la media masculina, especialmente en el caso de los docentes de Ciencias. Más adelante se analizarán estas diferencias por medio del coeficiente de correlación biserial puntual.

A continuación se da cuenta de los resultados obtenidos por el profesorado en cada una de las preguntas del cuestionario de conceptos sobre astronomía, 
CONOCIMIENTOS ASTRONÓMICOS DEL PROFESORADO DE EDUCACIÓN SECUNDARIA OBLIGATORIA Y PREFERENCIAS METODOLÓGICAS PARA LA ENSEÑANZA DE ASTRONOMÍA

complementando los mismos con el detalle en función de si la especialidad de los docentes correspondía o no a materias científicas. En las tablas se muestra en negrita la respuesta correcta. En el caso de los gráficos, la respuesta correcta corresponde a la columna sombreada en gris.

Abordaremos en primer lugar las cuestiones referidas a las estaciones del año y la duración del día y la noche. En primer lugar, el 86\% de los profesores tiene claro que existen dos días al año en los que día y noche tienen la misma duración (equinoccios), aunque conviene reseñar que un 20\% del profesorado "no científico" contestó que esta circunstancia se da sólo un día al año.

\begin{tabular}{|l|c|c|c|c|c|}
\hline \multicolumn{5}{|c|}{ ¿HAY ALGÚn DÍA DEL AÑo EN QUE LA DURACIÓN DEL DÍA SEA IGUAL A LA DE LA NOCHE? } \\
\hline & Ninguno & Uno & Dos & Más de dos & Ns/Nc \\
\hline Ciencias & $0 \%$ & $10 \%$ & $90 \%$ & $0 \%$ & $0 \%$ \\
\hline No Ciencias & $0 \%$ & $20 \%$ & $70 \%$ & $0 \%$ & $10 \%$ \\
\hline
\end{tabular}

Por otra parte, sólo el 20\% de los participantes identificó que un día con 14 horas de luz puede darse tanto en primavera como en verano, apreciándose que incluso la mitad del profesorado de Ciencias asoció tal circunstancia exclusivamente al verano. El hecho de asociar mayores horas de luz con el verano es una creencia muy extendida, cuando en realidad la longitud de los días crece en primavera y decrece en verano.

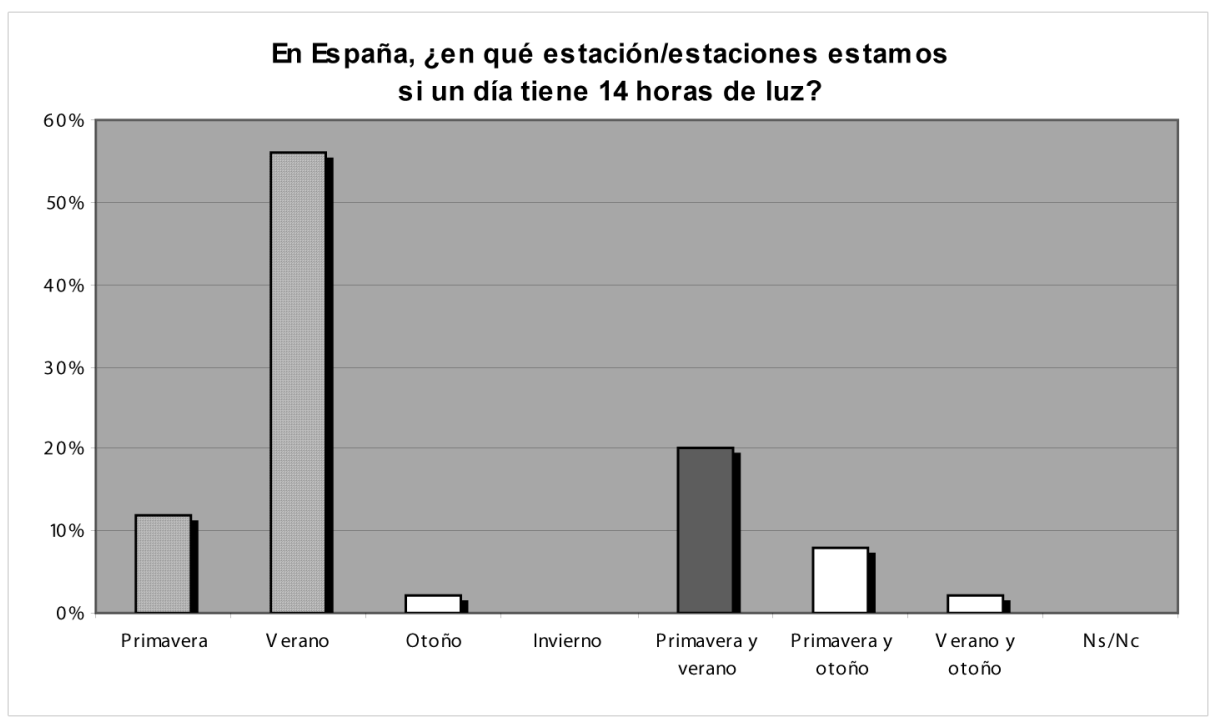


JOSÉ LUIS GARCÍA HERRERO

CONOCIMIENTOS ASTRONÓMICOS DEL PROFESORADO DE EDUCACIÓN SECUNDARIA OBLIGATORIA Y PREFERENCIAS METODOLÓGICAS PARA LA ENSEÑANZA DE ASTRONOMÍA

\begin{tabular}{|l|c|c|c|c|}
\hline \multicolumn{5}{|c|}{ EN ESPAÑA, SI UN DÍA TIENE 14 HORAS DE LUZ, ¿EN QUÉ ESTACIÓN (O ESTACIONES) } \\
& NOS ENCONTRAMOS? & Otoño & Invierno \\
\hline & Primavera & Verano & $0 \%$ & $0 \%$ \\
\hline Ciencias & $12,5 \%$ & $50 \%$ & $10 \%$ & $0 \%$ \\
\hline No Ciencias & $10 \%$ & $80 \%$ & Verano y otoño & Ns/Nc \\
\hline & Primavera y verano & Primavera y otoño & $2,5 \%$ & $0 \%$ \\
\hline Ciencias & $25 \%$ & $10 \%$ & $0 \%$ & $0 \%$ \\
\hline No Ciencias & $0 \%$ & $0 \%$ & & \\
\hline
\end{tabular}

Al preguntar directamente por la causa de las estaciones del año, se obtuvo un $74 \%$ de respuestas correctas, pero es muy sorprendente que un porcentaje muy considerable de docentes contestara que el Sol se mueve alrededor de la Tierra. Parece lógico considerar que tal respuesta sólo puede ser indicativa de falta de concentración al contestar el cuestionario.

\begin{tabular}{|l|c|c|c|c|c|}
\hline & $\begin{array}{c}\text { ¿POR QUÉ EXISTEN LAS ESTACIONES DEL AÑO? } \\
\text { alrededor de } \\
\text { la Tierra }\end{array}$ & $\begin{array}{c}\text { Inclinación } \\
\text { eje rotación } \\
\text { terrestre }\end{array}$ & $\begin{array}{c}\text { Movimiento } \\
\text { Sol alrededor } \\
\text { Tierra }\end{array}$ & $\begin{array}{c}\text { Cambios } \\
\text { distancia } \\
\text { Tierra-Sol }\end{array}$ & Ns/Nc \\
\hline Ciencias & $0 \%$ & $\mathbf{8 2 , 5 \%}$ & $17,5 \%$ & $0 \%$ & $0 \%$ \\
\hline No Ciencias & $0 \%$ & $\mathbf{4 0 \%}$ & $50 \%$ & $0 \%$ & $10 \%$ \\
\hline
\end{tabular}

Aunque al preguntar indirectamente por la causa de las estaciones haciendo referencia al calor del verano, se alcanza un $76 \%$ de respuestas correctas, el profesorado de materias no científicas se hace eco de una concepción alternativa muy frecuente: considerar que las estaciones del año están causadas por la variación en la distancia Tierra-Sol, con un 50\% de respuestas en este sentido. El hecho de que en la pregunta anterior ningún profesor justificara la existencia de las estaciones por los cambios en la distancia Tierra-Sol parecen indicar bastante confusión con respecto a la comprensión del fenómeno de las estaciones por parte del profesorado no científico.

\begin{tabular}{|l|c|c|c|c|c|}
\hline \multicolumn{7}{|c|}{ ¿POR QUÉ CREE QUE HACE MÁS CALOR EN VERANO? } \\
\hline & $\begin{array}{c}\text { Tierra más } \\
\text { cerca del Sol }\end{array}$ & $\begin{array}{c}\text { Eje terrestre } \\
\text { inclinado } \\
\text { hacia el Sol }\end{array}$ & $\begin{array}{c}\text { Menos nubes } \\
\text { y lluvia en } \\
\text { verano }\end{array}$ & $\begin{array}{c}\text { Menos horas } \\
\text { de luz en } \\
\text { verano }\end{array}$ & Ns/Nc \\
\hline Ciencias & $5 \%$ & $\mathbf{9 0 \%}$ & $2,5 \%$ & $2,5 \%$ & $0 \%$ \\
\hline No Ciencias & $50 \%$ & $\mathbf{2 0} \%$ & $0 \%$ & $10 \%$ & $20 \%$ \\
\hline
\end{tabular}



Y PREFERENCIAS METODOLÓGICAS PARA LA ENSEÑANZA DE ASTRONOMÍA

En concordancia con las cuestiones anteriores, y de forma extensible a los docentes de todas las especialidades, existe un gran desconocimiento de la forma real de la órbita terrestre, ya que la enorme mayoría la asocia con una elipse excéntrica, en vez de con una forma prácticamente circular. Es muy probable que la explicación de tal desconocimiento radique en que las ilustraciones que aparecen en los libros suelen mostrarse en perspectiva lateral y no cenital, haciendo que la órbita circular aparezca con forma de elipse.

\begin{tabular}{|l|c|c|c|}
\hline \multicolumn{4}{|c|}{ ¿CuÁL DE LOS SIGUIENTES DibujOs MUESTRA CON MAYOR EXACTITUD } \\
& LA ÓRBITA DE LA TIERRA ALREDEDOR DEL SOL? & \multirow{2}{*}{ Ns/Nc } \\
& Elipse excéntrica & Círculo & \\
\hline Ciencias & & $\mathbf{1 7 , 5 \%}$ & $0 \%$ \\
\hline No Ciencias & $82,5 \%$ & $\mathbf{0} \%$ & $20 \%$ \\
\hline
\end{tabular}

Nos centramos ahora en el bloque de cuestiones referidas a la Luna, el Sol y sus posiciones relativas en las fases lunares y eclipses.

En primer lugar, el 78\% del profesorado parece tener claro que la sincronía existente entre los movimientos de rotación y traslación lunar es la responsable de que sólo podamos ver la mitad de la superficie lunar. Sin embargo, dos de cada tres profesores no científicos muestran su desconocimiento al respecto, e incluso la mitad piensa que la Luna no rota sobre su eje.

\begin{tabular}{|l|c|c|c|c|c|}
\hline & $\begin{array}{c}\text { Luna no rota } \\
\text { sobre su eje }\end{array}$ & $\begin{array}{c}\text { ¿Pismos } \\
\text { períodos de } \\
\text { traslación y } \\
\text { rotación }\end{array}$ & $\begin{array}{c}\text { Vemos } \\
\text { superficie } \\
\text { completa }\end{array}$ & $\begin{array}{c}\text { Luna no } \\
\text { se traslada } \\
\text { alrededor de } \\
\text { la Tierra }\end{array}$ & Ns/NC \\
\hline Ciencias & $10 \%$ & $90 \%$ & $0 \%$ & $0 \%$ & $0 \%$ \\
\hline No Ciencias & $50 \%$ & $30 \%$ & $10 \%$ & $0 \%$ & $10 \%$ \\
\hline
\end{tabular}

En relación a la duración de las fases lunares, la enorme mayoría de los docentes tiene claro que éstas duran aproximadamente un mes.

EL JEFE INDIO HA DICHO: “MI HIJA CUMPLE HOY DIEZ LUNAS" ¿QUÉ EDAD TIENE SU HIJA?

\begin{tabular}{|l|c|c|c|c|c|}
\hline & 10 días & 10 semanas & 10 meses & 10 años & Ns/Nc \\
\hline Ciencias & $0 \%$ & $7,5 \%$ & $92,5 \%$ & $0 \%$ & $0 \%$ \\
\hline No Ciencias & $10 \%$ & $0 \%$ & $90 \%$ & $0 \%$ & $0 \%$ \\
\hline
\end{tabular}


CONOCIMIENTOS ASTRONÓMICOS DEL PROFESORADO DE EDUCACIÓN SECUNDARIA OBLIGATORIA Y PREFERENCIAS METODOLÓGICAS PARA LA ENSEÑANZA DE ASTRONOMÍA

Al plantear las siguientes dos preguntas sobre la posición relativa Sol-TierraLuna, era de esperar que los porcentajes de respuesta no fueran demasiado buenos, ya que se hace necesario el razonamiento espacial para asociar el aspecto de la Luna con la posición relativa de los tres astros. Sin embargo, el porcentaje de aciertos de la siguiente pregunta fue aceptable, situándose en el 64\%, e incluso bastante mejor en el caso de los profesores de disciplinas no científicas. Quizás pueda achacarse a que el profesorado haya experimentado por sí mismo la respuesta correcta al haber contemplado alguna vez una puesta de Sol al mismo tiempo que la Luna llena aparece por el horizonte opuesto.

SI OBSERVA LA LUNA LLENA SALIR POR EL ESTE; ¿¿UUÉ HORA DEL DÍA CREE QUE PUEDE SER?

\begin{tabular}{|l|c|c|c|c|c|}
\hline & Amanecer & Atardecer & Mediodía & Medianoche & Ns/Nc \\
\hline Ciencias & $10 \%$ & $\mathbf{6 0} \%$ & $2,5 \%$ & $7,5 \%$ & $20 \%$ \\
\hline No Ciencias & $0 \%$ & $\mathbf{8 0} \%$ & $0 \%$ & $10 \%$ & $10 \%$ \\
\hline
\end{tabular}

El porcentaje de acierto descendió hasta el 42\% en la siguiente pregunta, ya que el razonamiento espacial asociado no es trivial. Casi el 50\% del profesorado de Ciencias contestó correctamente, frente al 20\% del resto de docentes:
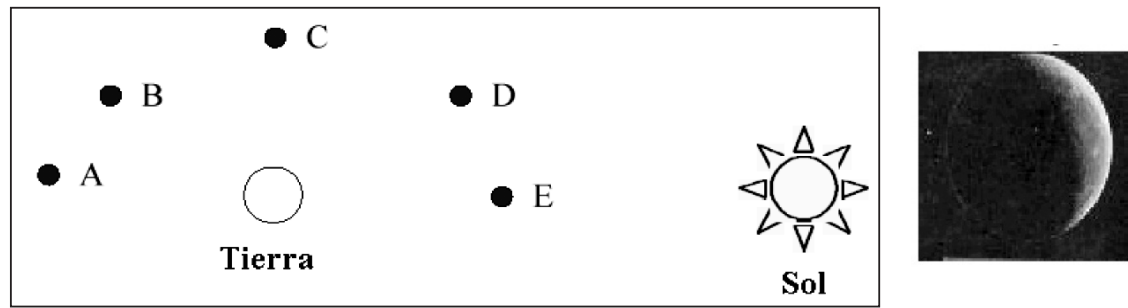

EN LA SITUACIÓN EXPLICADA EN LA IMAGEN ANTERIOR; ¿QUÉ POSICIÓN DE LA LUNA CAUSARÍA QUE ÉSTA SE OBSERVASE DESDE LA TIERRA COMO EN LA FOTOGRAFÍA?

\begin{tabular}{|l|c|c|c|c|c|c|}
\hline & $\begin{array}{c}\text { Luna } \\
\text { llena } \\
\text { (A) }\end{array}$ & $\begin{array}{c}\text { Entre llena y } \\
\text { cuarto creciente } \\
\text { (B) }\end{array}$ & $\begin{array}{c}\text { Cuarto } \\
\text { creciente } \\
\text { (C) }\end{array}$ & $\begin{array}{c}\text { Entre nueva } \\
\text { y cuarto } \\
\text { creciente (D) }\end{array}$ & $\begin{array}{c}\text { Luna } \\
\text { nueva } \\
\text { (E) }\end{array}$ & Ns/Nc \\
\hline Ciencias & $10 \%$ & $22,5 \%$ & $5 \%$ & $\mathbf{4 7 , 5} \%$ & $2,5 \%$ & $12,5 \%$ \\
\hline No Ciencias & $20 \%$ & $20 \%$ & $30 \%$ & $\mathbf{2 0} \%$ & $10 \%$ & $0 \%$ \\
\hline
\end{tabular}

El razonamiento de las cuestiones referidas a la situación relativa Sol-TierraLuna se torna más sencillo en el caso de los eclipses, aunque en el caso del eclipse de Sol los resultados muestran que existe cierta confusión sobre si se da en Luna llena o Luna nueva, incluso entre el profesorado de Ciencias. El porcentaje global de acierto en este caso fue del 60\%, aunque cerca del 30\% de los docentes asociaron el eclipse de Sol con la Luna llena. Como se puede apreciar, estos 
CONOCIMIENTOS ASTRONÓMICOS DEL PROFESORADO DE EDUCACIÓN SECUNDARIA OBLIGATORIA Y PREFERENCIAS METODOLÓGICAS PARA LA ENSEÑANZA DE ASTRONOMÍA

porcentajes prácticamente se invierten cuando se consideran en función de la especialidad del profesorado.

\begin{tabular}{|l|c|c|c|c|c|}
\hline \multirow{2}{*}{ ¿EN QUÉ FASE DEBE ENCONTRARSE LA LUNA PARA QUE SE PUEDA PRODUCIR UN ECLIPSE DE SOL? } \\
\hline Luna nueva & Luna llena & $\begin{array}{c}\text { Cuarto } \\
\text { menguante }\end{array}$ & $\begin{array}{c}\text { Cuarto } \\
\text { creciente }\end{array}$ & Ns/Nc \\
\hline Ciencias & $\mathbf{7 0} \%$ & $20 \%$ & $2,5 \%$ & $0 \%$ & $7,5 \%$ \\
\hline No Ciencias & $\mathbf{2 0} \%$ & $60 \%$ & $0 \%$ & $0 \%$ & $20 \%$ \\
\hline
\end{tabular}

En el caso de los eclipses de Luna, el porcentaje de acierto fue mucho mayor, alcanzando prácticamente el $80 \%$. Sin embargo, es muy sorprendente y desconcertante que un 6\% del profesorado contestara que el Sol se puede situar entre la Tierra y la Luna.

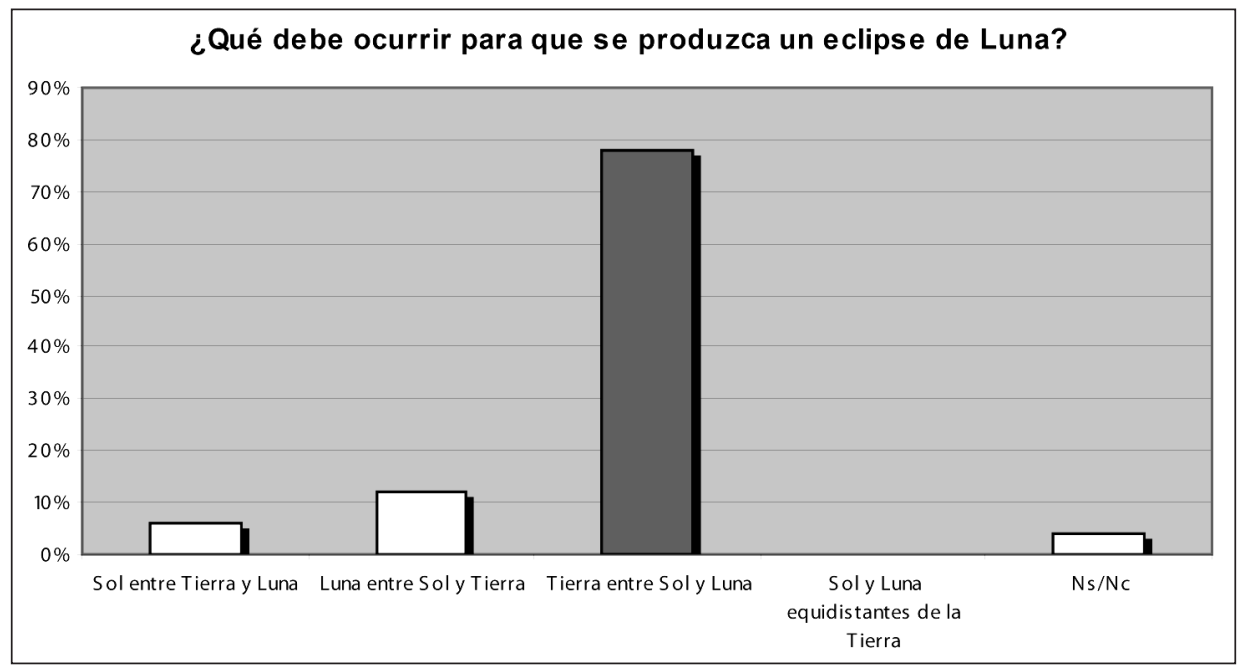

\begin{tabular}{|l|c|c|c|c|c|}
\hline \multicolumn{7}{|c|}{ ¿QUÉ DEBE OCURRIR PARA QUE SE PRODUZCA UN ECLIPSE DE LUNA? } \\
\hline & $\begin{array}{c}\text { Sol entre } \\
\text { Tierra y Luna }\end{array}$ & $\begin{array}{c}\text { Luna entre } \\
\text { Sol y Tierra }\end{array}$ & $\begin{array}{c}\text { Tierra entre } \\
\text { Sol y Luna }\end{array}$ & $\begin{array}{c}\text { Sol y Luna a igual } \\
\text { distancia de la Tierra }\end{array}$ & Ns/Nc \\
\hline Ciencias & $5 \%$ & $2,5 \%$ & $90 \%$ & $0 \%$ & $2,5 \%$ \\
\hline No Ciencias & $10 \%$ & $50 \%$ & $30 \%$ & $0 \%$ & $10 \%$ \\
\hline
\end{tabular}

Sobre los movimientos del Sol, debemos diferenciar entre sus movimientos propios de rotación y traslación, y su movimiento aparente en el cielo debido a la rotación terrestre. Se incluyó en el cuestionario una pregunta de cada tipo. Para 
CONOCIMIENTOS ASTRONÓMICOS DEL PROFESORADO DE EDUCACIÓN SECUNDARIA OBLIGATORIA Y PREFERENCIAS METODOLÓGICAS PARA LA ENSEÑANZA DE ASTRONOMÍA

la pregunta del primer aspecto, el porcentaje global de acierto fue del $82 \%$, con diferencias notables entre ambos tipos de profesorado.

\begin{tabular}{|l|c|c|c|c|c|}
\hline \multicolumn{7}{|c|}{ LOS MOVIMIENTOS DEL SOL SON } \\
\hline Rotación & $\begin{array}{c}\text { Reposo } \\
\text { absoluto }\end{array}$ & $\begin{array}{c}\text { Traslación } \\
\text { alrededor } \\
\text { Tierra }\end{array}$ & $\begin{array}{c}\text { Rotación sobre su eje } \\
\text { y traslación alrededor } \\
\text { centro Vía Láctea }\end{array}$ & Ns/Nc \\
\hline Ciencias & $0 \%$ & $7,5 \%$ & $0 \%$ & $90 \%$ & $2,5 \%$ \\
\hline No Ciencias & $20 \%$ & $30 \%$ & $0 \%$ & $50 \%$ & $0 \%$ \\
\hline
\end{tabular}

El porcentaje de acierto desciende notablemente en el caso de la pregunta relativa al movimiento aparente del Sol, ya que se introdujo como factor de distracción el fondo estelar. Tan sólo se obtuvo un 22\% de aciertos, mientras que el 52\% de las respuestas se otorgaron a la constelación de Piscis, lo que muestra claramente la dificultad para percibir el Sol como «una estrella más» del firmamento, al atribuirle movimiento aparente propio, en detrimento del resto de estrellas del firmamento. Es decir, se tiene claro el movimiento aparente individual del Sol, pero no como parte del movimiento de la bóveda celeste completa.

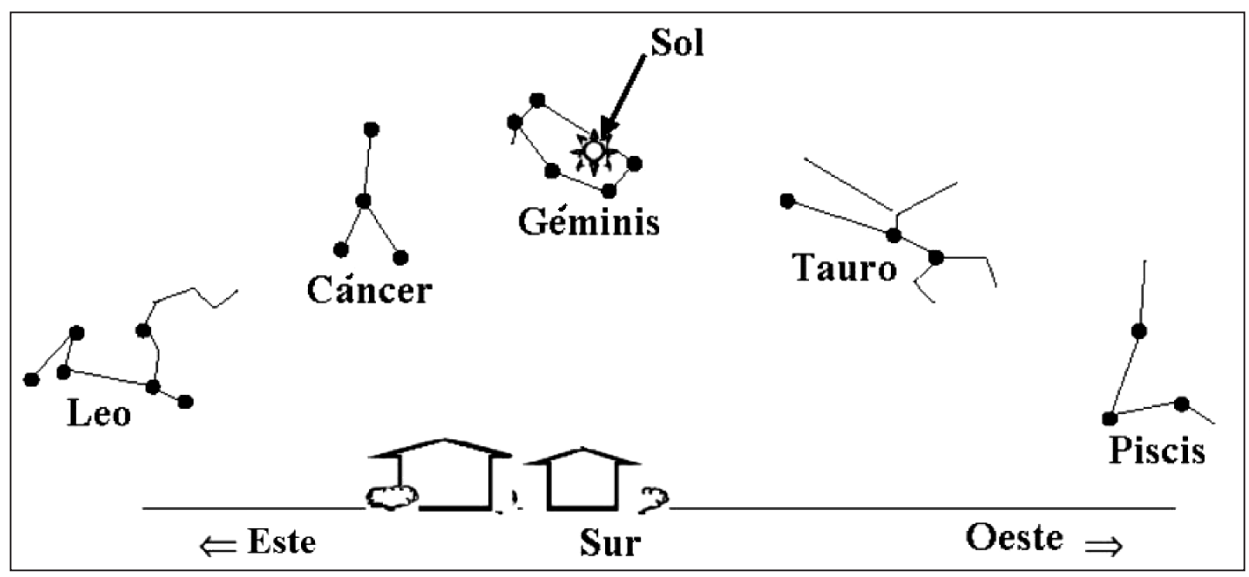

EN LA SITUACIÓN EXPLICADA POR LA IMAGEN ANTERIOR; ¿CERCA DE QUÉ CONSTELACIÓN ESTARÍA SITUADO EL SOL AL ATARDECER?

\begin{tabular}{|l|c|c|c|c|c|c|}
\hline & Leo & Cáncer & Géminis & Tauro & Piscis & Ns/Nc \\
\hline Ciencias & $2,5 \%$ & $10 \%$ & $\mathbf{2 5} \%$ & $10 \%$ & $45 \%$ & $7,5 \%$ \\
\hline No Ciencias & $10 \%$ & $0 \%$ & $\mathbf{1 0} \%$ & $0 \%$ & $80 \%$ & $0 \%$ \\
\hline
\end{tabular}



Y PREFERENCIAS METODOLÓGICAS PARA LA ENSEÑANZA DE ASTRONOMÍA

Otra cuestión relevante es la altura que alcanza el Sol en el firmamento, la cual depende exclusivamente de la latitud del punto de observación. La cuestión reservada a este aspecto en el cuestionario también muestra confusión y desconocimiento al respecto, siendo similares los porcentajes de acierto por especialidad. Sólo en las latitudes comprendidas entre los trópicos, el Sol llega a situarse en el cenit celeste, lo cual ocurre dos veces al año. A pesar de que la mayoría de las respuestas son correctas, situándose los aciertos en el 40\%, un tercio de los encuestados muestran la creencia de que el Sol al mediodía se sitúa en el cenit celeste, confundiendo el hecho cierto de que al mediodía el Sol alcanza su altura máxima.

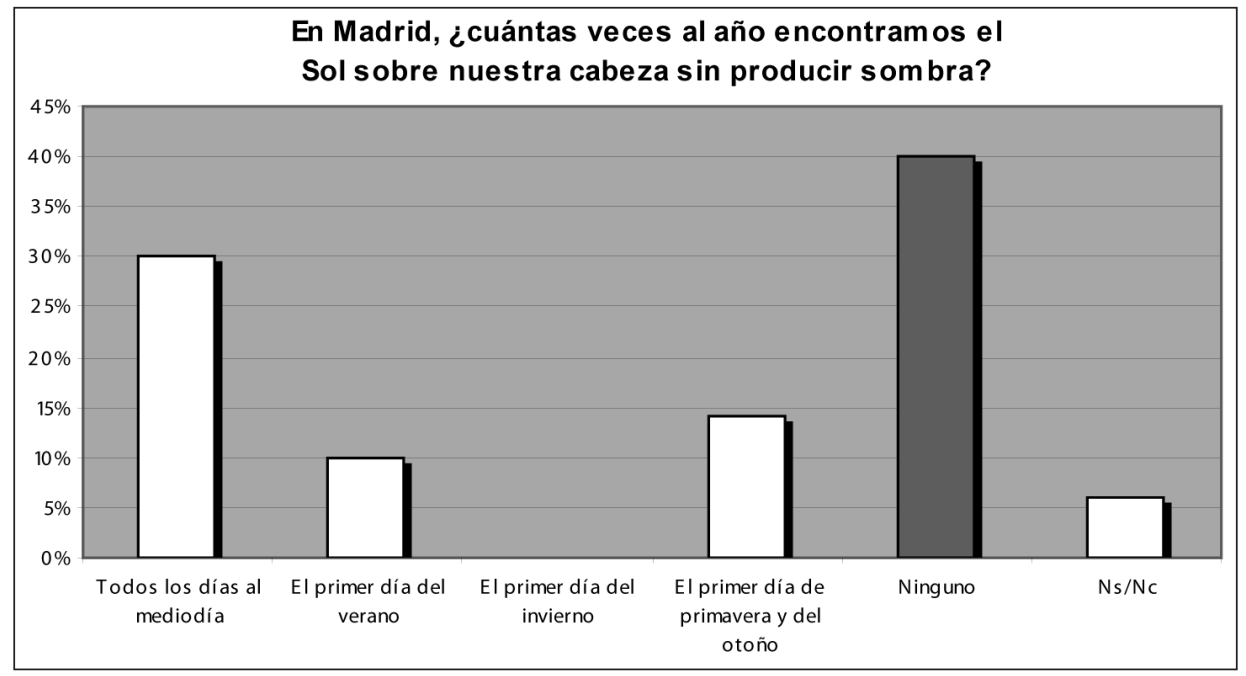

\begin{tabular}{|l|c|c|c|c|c|c|}
\hline \multicolumn{6}{|c|}{ EN MADRID, ¿CUÁNTAS VECES AL AÑO ESTÁ EL SOL DIRECTAMENTE SOBRE NUESTRA CABEZA } \\
\hline & $\begin{array}{c}\text { Todos los } \\
\text { días al } \\
\text { mediodía }\end{array}$ & $\begin{array}{c}\text { El primer } \\
\text { día del } \\
\text { verano }\end{array}$ & $\begin{array}{c}\text { El primer } \\
\text { día del } \\
\text { invierno }\end{array}$ & $\begin{array}{c}\text { El primer día } \\
\text { de primavera } \\
\text { y otoño }\end{array}$ & Ninguno & Ns/Nc \\
\hline Ciencias & $32,5 \%$ & $12,5 \%$ & $0 \%$ & $10 \%$ & $40 \%$ & $5 \%$ \\
\hline No Ciencias & $20 \%$ & $0 \%$ & $0 \%$ & $30 \%$ & $40 \%$ & $10 \%$ \\
\hline
\end{tabular}

Diversos aspectos sobre la estrella polar conformaron otra de las preguntas del cuestionario, siendo los porcentajes de acierto bastante elevados en todos los casos. Sólo es reseñable que un 40\% del profesorado de materias no científicas incluyó a la estrella polar dentro del asterismo de la Osa Mayor, cuando en realidad pertenece a la Osa Menor. Tal circunstancia pone a su vez de relieve la dificultad del profesorado, como cualquier otra persona en general, para ubicarla en el cielo. 
CONOCIMIENTOS ASTRONÓMICOS DEL PROFESORADO DE EDUCACIÓN SECUNDARIA OBLIGATORIA Y PREFERENCIAS METODOLÓGICAS PARA LA ENSEÑANZA DE ASTRONOMÍA

En el siguiente gráfico se pueden apreciar los porcentajes globales de acierto y error para las diversas afirmaciones sobre la estrella polar, así como los porcentajes de acierto por especialidad en la tabla que lo acompaña.

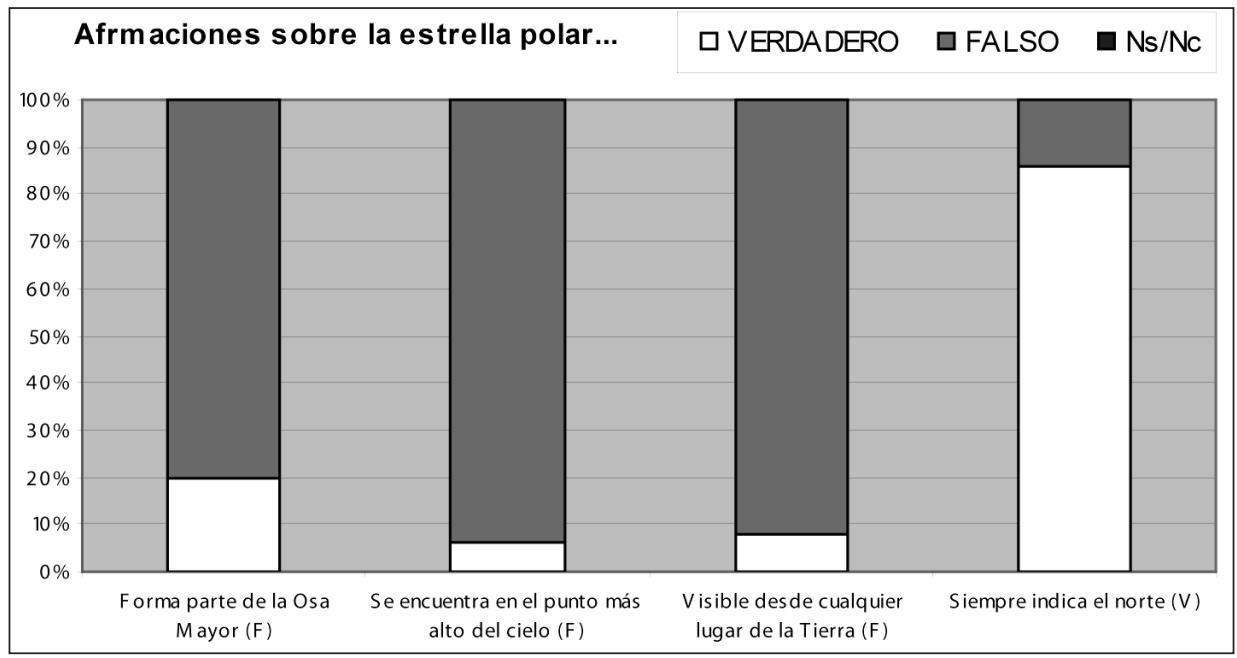

\begin{tabular}{|l|c|c|c|c|}
\hline \% ACIERTO & $1 .^{a}$ AFIRMACIÓN & 2. ${ }^{\text {a }}$ AFIRMACIÓN & 3. ${ }^{\text {a }}$ AFIRMACIÓN & $4 .{ }^{a}$ AFIRMACIÓN \\
\hline Ciencias & $85 \%$ & $92,5 \%$ & $92,5 \%$ & $87,5 \%$ \\
\hline No Ciencias & $60 \%$ & $100 \%$ & $90 \%$ & $80 \%$ \\
\hline
\end{tabular}

Con relación a los movimientos de los planetas por la bóveda celeste, el 78\% de los profesores participantes conoce que los planetas se desplazan a lo largo de la eclíptica, pero la mayoría desconoce la verdadera relevancia de las constelaciones del zodíaco, que son aquellas por las que discurre la eclíptica. Prácticamente la misma proporción de los encuestados piensan que estas constelaciones contienen las estrellas más cercanas y que son parte del sendero por el que discurren los planetas. El desconocimiento al respecto es compartido en igual medida por los docentes, independientemente de su especialidad.

\begin{tabular}{|c|c|c|c|c|c|}
\hline \multicolumn{6}{|c|}{$\begin{array}{l}\text { ¿CÓMO SE DENOMINA LA FRANJA DEL CIELO POR LA QUE SE DESPLAZAN EL SOL, } \\
\text { LA LUNA Y LOS PLANETAS? }\end{array}$} \\
\hline & Meridiano & Vía Láctea & Eclíptica & Camino Santiago & $\mathrm{Ns} / \mathrm{Nc}$ \\
\hline Ciencias & $2,5 \%$ & $15 \%$ & $77,5 \%$ & $0 \%$ & $5 \%$ \\
\hline No Ciencias & $0 \%$ & $10 \%$ & $80 \%$ & $0 \%$ & $10 \%$ \\
\hline
\end{tabular}


JOSÉ LUIS GARCÍA HERRERO

CONOCIMIENTOS ASTRONÓMICOS DEL PROFESORADO DE EDUCACIÓN SECUNDARIA OBLIGATORIA Y PREFERENCIAS METODOLÓGICAS PARA LA ENSEÑANZA DE ASTRONOMÍA

\begin{tabular}{|l|c|c|c|c|c|}
\hline \multicolumn{7}{|c|}{ ¿CUÁL ES LA IMPORTANCIA DE LAS CONSTELACIONES DEL ZODÍACO? } \\
\hline $\begin{array}{c}\text { Determinan } \\
\text { destino }\end{array}$ & $\begin{array}{c}\text { Contienen } \\
\text { estrellas más } \\
\text { cercanas }\end{array}$ & $\begin{array}{c}\text { Sendero de los } \\
\text { planetas }\end{array}$ & $\begin{array}{c}\text { Las más } \\
\text { extensas }\end{array}$ & Ns/Nc \\
\hline Ciencias & $2,5 \%$ & $40 \%$ & $42,5 \%$ & $2,5 \%$ & $12,5 \%$ \\
\hline No Ciencias & $0 \%$ & $40 \%$ & $40 \%$ & $0 \%$ & $20 \%$ \\
\hline
\end{tabular}

El cuestionario incluía una pregunta sobre la identificación del asterismo de la constelación de Orión, a la que el 70\% de los docentes contestó correctamente, sin diferencias llamativas en función de la especialidad.

\begin{tabular}{|l|c|c|c|c|c|}
\hline \multicolumn{7}{|c|}{ IDENTIFIQUE LA CONSTELACIÓN DE ORIÓN } \\
\hline & Leo & Sagitario & Escorpión & Orión & Ns/Nc \\
\hline Ciencias & $7,5 \%$ & $7,5 \%$ & $2,5 \%$ & $70 \%$ & $12,5 \%$ \\
\hline No Ciencias & $10 \%$ & $10 \%$ & $0 \%$ & $70 \%$ & $10 \%$ \\
\hline
\end{tabular}

La última pregunta versó sobre Cosmología, y la enorme mayoría de los encuestados contestó correctamente:

\begin{tabular}{|l|c|c|c|c|}
\hline \multicolumn{5}{|c|}{ ¿CUÁL ES EL ESTADO ACTUAL DEL UNIVERSO? } \\
\hline & $\begin{array}{c}\text { Expansión con atracción de } \\
\text { objetos cercanos }\end{array}$ & Contracción & Reposo & Ns/Nc \\
\hline Ciencias & $\mathbf{1 0 0} \%$ & $0 \%$ & $0 \%$ & $0 \%$ \\
\hline No Ciencias & $\mathbf{8 0} \%$ & $10 \%$ & $10 \%$ & $0 \%$ \\
\hline
\end{tabular}

\subsection{Comparativa de resultados}

Continuando con el análisis de resultados sobre conceptos de astronomía, se recurrió al cálculo del coeficiente de correlación biserial-puntual $\left(\mathrm{r}_{\mathrm{bp}}\right)$ para estudiar el grado de relación existente entre la puntuación obtenida y diversas variables dicotómicas (Pérez et al., 2009).

Como se ha explicado anteriormente, los alumnos también respondieron un cuestionario de conceptos astronómicos que incluía las preguntas respondidas por el profesorado, por lo que fue posible estudiar la correlación entre las puntuaciones obtenidas por ambos grupos. Para el cálculo de la puntuación media del alumnado y su posterior comparación con el profesorado, sólo se han tenido en cuenta las preguntas comunes con los docentes, lo cual ha hecho que la media de la puntuación sobre 10 del alumnado bajara de 4,2 a 3,5. 
CONOCIMIENTOS ASTRONÓMICOS DEL PROFESORADO DE EDUCACIÓN SECUNDARIA OBLIGATORIA Y PREFERENCIAS METODOLÓGICAS PARA LA ENSEÑANZA DE ASTRONOMÍA

A continuación se muestra una tabla con las variables analizadas y los coeficientes obtenidos.

\begin{tabular}{|l|l|l|}
\hline \multicolumn{1}{|c|}{ VARIABles } & $\begin{array}{c}\text { PUNTUACIÓN MEDIA } \\
\text { SOBRE } 10\end{array}$ & $\mathrm{R}_{\mathrm{BP}}$ \\
\hline Profesor Ciencias/Alumno & $7,0 / 3,5$ & 0,65 \\
\hline Profesor No Ciencias/Alumno & $4,8 / 3,5$ & 0,17 \\
\hline Especialidad Profesorado Ciencias/No Ciencias & $7,0 / 4,8$ & 0,52 \\
\hline Género profesorado V/M & $7,1 / 5,7$ & 0,45 \\
\hline Género profesorado de Ciencias V/M & $7,4 / 6,4$ & 0,40 \\
\hline
\end{tabular}

Teniendo en cuenta los intervalos de categorización establecidos por Pérez et al. (2009), podemos concluir que, como era de esperar, existe una correlación notable entre las puntuaciones obtenidas por el profesorado de Ciencias y el alumnado $(0,65)$. Sin embargo, es muy llamativo que se obtiene una correlación muy baja cuando consideramos las puntuaciones del profesorado de especialidades no científicas y el alumnado $(0,17)$, de donde se desprende que no hay una relación muy marcada entre ambas variables.

Además, se aprecia una marcada correlación al considerar las puntuaciones obtenidas en función de la especialidad, científica o no, del profesorado $(0,52)$.

Si se considera el sexo del profesorado, se llega a la conclusión de que existe una correlación media $(0,45)$, siendo superiores las puntuaciones de los varones. La correlación es algo más baja cuando se considera exclusivamente el sexo del profesorado de Ciencias $(0,40)$.

\subsection{Metodologías de enseñanza-aprendizaje}

El diseño del cuestionario incluyó en su parte final una pregunta sobre las preferencias metodológicas en la enseñanza-aprendizaje de la astronomía, con 15 propuestas metodológicas en forma de escala de Likert de 5 puntos, a valorar desde "muy en desacuerdo" (1) hasta "muy de acuerdo" (5). En la elección de las metodologías propuestas se intentó aunar el aspecto pragmático de su aplicación real en el aula y la presencia de diferentes enfoques estratégicos, dentro del espectro de los enfoques mencionados en el apartado 2.

La siguiente tabla muestra los resultados por orden de preferencia para profesores y alumnos: 
CONOCIMIENTOS ASTRONÓMICOS DEL PROFESORADO DE EDUCACIÓN SECUNDARIA OBLIGATORIA Y PREFERENCIAS METODOLÓGICAS PARA LA ENSEÑANZA DE ASTRONOMÍA

\begin{tabular}{|c|c|c|c|}
\hline \multicolumn{4}{|c|}{$\begin{array}{l}\text { PREFERENCIAS METODOLÓGICAS EN LA ENSEÑANZA-APRENDIZAJE DE ASTRONOMÍA } \\
\text { (VALORACIÓN DE } 1 \text { A } 5 \text { ) }\end{array}$} \\
\hline PROFESORES & MEDIA & ALUMNOS & MEDIA \\
\hline $\begin{array}{l}\text { Observación telescopios/ } \\
\text { prismáticos }\end{array}$ & 4,74 & Visita al planetario & 4,29 \\
\hline Visita al planetario & 4,63 & $\begin{array}{l}\text { Observación telescopios/ } \\
\text { prismáticos }\end{array}$ & 4,20 \\
\hline Trabajos de investigación & 4,56 & Documentales & 3,94 \\
\hline Programas informáticos astronomía & 4,44 & Trabajos de investigación & 3,76 \\
\hline Prensa escrita & 4,36 & Debates & 3,74 \\
\hline Maquetas, relojes de sol... & 4,31 & Programas informáticos astronomía & 3,74 \\
\hline Charlas astrónomos profesionales & 4,30 & Charlas astrónomos profesionales & 3,67 \\
\hline Documentales & 4,28 & Maquetas, relojes de sol... & 3,52 \\
\hline Exposiciones orales pareja o grupo & 4,00 & Lección magistral & 3,24 \\
\hline Debates & 3,96 & Trabajos escritos en pareja o grupo & 3,08 \\
\hline Exposiciones orales individuales & 3,90 & Prensa escrita & 3,08 \\
\hline Trabajos escritos en pareja o grupo & 3,52 & Películas ciencia-ficción & 3,07 \\
\hline Trabajos escritos individuales & 3,28 & Exposiciones orales pareja o grupo & 2,98 \\
\hline Películas ciencia-ficción & 3,20 & Exposiciones orales individuales & 2,67 \\
\hline Lección magistral & 3,10 & Trabajos escritos individuales & 2,60 \\
\hline
\end{tabular}

En primer lugar, se constata que las puntuaciones asignadas por los alumnos son por lo general más bajas, por lo que éstos parecen valorar en menor medida que el profesorado las metodologías propuestas.

Centrándonos en la comparación de resultados, es muy clara la coincidencia en las dos metodologías más valoradas por ambos grupos: la visita al planetario y la observación del cielo con prismáticos y telescopio. También se valora positivamente por ambas partes la realización de trabajos de investigación y el uso de programas informáticos relacionados con la astronomía.

Los trabajos escritos y exposiciones de carácter individual son las metodologías menos valoradas por los alumnos, situándose también entre las menos valoradas por el profesorado. Por último, llama la atención que la lección magistral sea la metodología peor valorada por los profesores, ya que en la práctica docente es la más utilizada en el aula.

Un estudio comparativo más profundo de los resultados anteriores se llevó a cabo por medio del análisis de varianza, con el fin de comprobar la existencia de diferencias estadísticamente significativas entre los grupos considerados. En otras palabras, se comprueba si se puede considerar que las muestras estudiadas son aleatorias de la misma población. En nuestro caso, el análisis se plantea en función de las siguientes variables: 
JOSÉ LUIS GARCÍA HERRERO

CONOCIMIENTOS ASTRONÓMICOS DEL PROFESORADO DE EDUCACIÓN SECUNDARIA OBLIGATORIA Y PREFERENCIAS METODOLÓGICAS PARA LA ENSEÑANZA DE ASTRONOMÍA

\begin{tabular}{|c|c|}
\hline \multicolumn{2}{|c|}{ VARIABLES DEL ANÁLISIS DE VARIANZA } \\
\hline Profesorado & Alumnado \\
\hline Género & Género \\
\hline Especialidad & Población \\
\hline- & Bilingüismo \\
\hline \multicolumn{2}{|c|}{ Estatus educativo: Profesor/Alumno } \\
\hline
\end{tabular}

En los análisis de varianza del profesorado, la especialidad se refiere a la diferenciación entre profesores que imparten o no asignaturas científicas. En el caso del alumnado, la diferenciación por tipología de población se determinó en función de la densidad de población del municipio, calculada a partir de datos del Instituto Nacional de Estadística (INE, 2013):

\begin{tabular}{|c|c|c|}
\hline \multicolumn{3}{|c|}{ DENSIDAD DE POBLACIÓN APROXIMADA (HAB. $/ \mathrm{KM}^{2}$ ) } \\
\hline Navalcarnero & Getafe & Madrid \\
\hline 250 & 2.177 & 5.345 \\
\hline
\end{tabular}

Aunque Navalcarnero no se considera un municipio de entorno rural por presentar una densidad de población superior a 100 hab. $/ \mathrm{km}^{2}$, al realizar el análisis de varianza se ha comparado a los alumnos de Navalcarnero con los de Getafe y Madrid en conjunto, por presentar Navalcarnero una densidad de población mucho menor y una tradición marcadamente agrícola. Por otra parte, se ha realizado otro análisis de varianza teniendo en cuenta las preferencias metodológicas de los alumnos pertenecientes a una sección bilingüe inglesa frente al resto.

Por último, se llevó a cabo un análisis comparativo de las preferencias metodológicas mostradas por profesores y alumnos, siendo este caso el que arrojó un mayor número de diferencias estadísticamente significativas.

La aplicación del análisis de varianza requiere tres requisitos previos: uso de escalas de intervalo para la variable dependiente (como la escala Likert), distribución normal de la variable dependiente y homogeneidad de varianzas de las poblaciones (homocedasticidad). Sin embargo, Morales (2008) confirma la validez general del análisis de varianza excepto cuando concurran al menos dos de las siguientes circunstancias: muestras pequeñas (aproximadamente $\mathrm{N}<25$ ), muestras de tamaño muy desigual (por ejemplo, $\left(\mathrm{N}_{1}<3 \mathrm{~N}_{2}\right)$ o muestras con varianzas muy desiguales (siendo la homocedasticidad la condición más importante). En tal caso, se recomienda el uso de pruebas no paramétricas, como la U de Mann-Whitney. Por tanto, cuando se ha detectado la posibilidad de que se dieran dos o más de estas condiciones, se ha realizado un análisis previo de homocedasticidad por medio del test de Levene (por ser la prueba menos sensible a la ausencia de distribución normal), aplicando posteriormente el test de Mann-Whitney en caso de necesidad. 

Y PREFERENCIAS METODOLÓGICAS PARA LA ENSEÑANZA DE ASTRONOMÍA

A continuación se detallan los resultados estadísticamente significativos, estableciendo el nivel de confianza en el 95\% $(\alpha=0,05)$. Se proporcionan los resultados de los tests de Levene y Mann-Whitney, cuando no se satisfacen las condiciones del análisis de varianza, así como el tamaño del efecto (r), según Field (2009). Además, para el análisis de varianza, se proporcionan los valores de la razón $\mathrm{F}$ y el p-valor $(\mathrm{p}<\alpha)$, así como el tamaño del efecto $(\mathrm{d})$, según Cohen (1988). En todos los casos se proporcionan los valores medios de las variables analizadas, y recordamos que los tamaños de las muestras figuran en las tablas del apartado 3 .

Añadiremos que, atendiendo a las orientaciones de Cohen (1988) y Field (2009), consideraremos las siguientes magnitudes en el tamaño del efecto:

\begin{tabular}{|c|c|c|c|}
\hline MAGNITUD DEL TAMAÑO DEL EFECTO & PEQUEÑA & MEDIA & GRANDE \\
\hline $\mathrm{d}$ & 0,20 & 0,50 & 0,80 \\
\hline $\mathrm{r}$ & 0,10 & 0,30 & 0,50 \\
\hline
\end{tabular}

Como Hipótesis Nula, afirmamos que todas las muestras proceden de la misma población, por lo que se espera encontrar que sus medias no diferirán significativamente y sus diferencias se justifican por el error muestral.

En primer lugar, y como se puede observar en las tablas inferiores, debemos reseñar que los análisis de varianza realizados en función de variables asociadas al profesorado no desvelan apenas datos significativos, con la excepción de que sí existen diferencias estadísticamente significativas a favor de los docentes de materias no científicas en la propuesta de trabajos escritos individuales como metodología apropiada, con una magnitud media del tamaño del efecto asociado.

PROFESORES EN FUNCIÓN DEL GÉNERO

No se obtuvieron diferencias estadísticamente significativas en las preferencias metodológicas

PROFESORES EN FUNCIÓN DE LA ESPECIALIDAD (CIENTÍFICA-NO CIENTÍFICA)

\begin{tabular}{|l|c|c|c|c|c|c|c|}
\hline \multirow{2}{*}{$\begin{array}{c}\text { Metodologías para el } \\
\text { aprendizaje de astronomía }\end{array}$} & \multicolumn{2}{|c|}{$\begin{array}{c}\text { Test de } \\
\text { Levene }\end{array}$} & \multicolumn{3}{c|}{$\begin{array}{c}\text { Test de } \\
\text { Mann-Whitney }\end{array}$} & \multicolumn{3}{c|}{ Media } \\
\cline { 2 - 9 } & $\mathrm{F}$ & $\mathrm{p}$ & $\mathrm{U}$ & $\mathrm{p}$ & $\mathrm{r}$ & Ciencias & No Ciencias \\
\hline $\begin{array}{l}\text { Trabajos escritos } \\
\text { individuales }\end{array}$ & 0,194 & 0,662 & 108,5 & 0,017 & 0,337 & 3,150 & 3,800 \\
\hline
\end{tabular}


CONOCIMIENTOS ASTRONÓMICOS DEL PROFESORADO DE EDUCACIÓN SECUNDARIA OBLIGATORIA Y PREFERENCIAS METODOLÓGICAS PARA LA ENSEÑANZA DE ASTRONOMÍA

A continuación se muestran los resultados de los análisis asociados a las medias del alumnado:

\begin{tabular}{|c|c|c|c|c|c|}
\hline \multicolumn{7}{|c|}{ ALUMNOS EN FUNCIÓN DEL GÉNERO } \\
\hline \multirow{2}{*}{$\begin{array}{c}\text { Metodologías para el } \\
\text { aprendizaje de astronomía }\end{array}$} & \multicolumn{3}{|c|}{ Análisis de varianza } & \multicolumn{2}{c|}{ Media } \\
\cline { 2 - 6 } & $\mathrm{F}$ & $\mathrm{p}$ & $\mathrm{d}$ & Varones & Mujeres \\
\hline $\begin{array}{l}\text { Observaciones con } \\
\text { telescopio o prismáticos }\end{array}$ & 4,914 & 0,027 & 0,266 & 4,056 & 4,310 \\
\hline
\end{tabular}

En primer lugar, se constata que el análisis de varianza en función del género únicamente desvela una diferencia significativa a favor de las alumnas en cuanto a la consideración de las observaciones del cielo con prismáticos o telescopio, siendo pequeño el tamaño del efecto asociado.

\begin{tabular}{|c|c|c|c|c|c|}
\hline \multicolumn{6}{|c|}{ ALUMNOS EN FUNCIÓN DE LA POBLACIÓN } \\
\hline \multirow{3}{*}{$\begin{array}{l}\text { Metodologías para el } \\
\text { aprendizaje de astronomía }\end{array}$} & \multirow{2}{*}{\multicolumn{3}{|c|}{ Análisis de varianza }} & \multirow{2}{*}{\multicolumn{2}{|c|}{$\frac{\text { Media }}{\text { Densidad Población }}$}} \\
\hline & & & & & \\
\hline & $\mathrm{F}$ & $\mathrm{p}$ & $\mathrm{d}$ & Alta & Baja \\
\hline $\begin{array}{l}\text { Proyección de películas de } \\
\text { ciencia ficción }\end{array}$ & 4,658 & 0,032 & 0,259 & 2,904 & 3,248 \\
\hline Trabajos de investigación & 4,020 & 0,046 & 0,241 & 3,880 & 3,635 \\
\hline
\end{tabular}

En segundo lugar, al estudiar los contrastes en función de la tipología de la población, se constatan diferencias significativas con tamaños del efecto muy moderados en el uso de películas de ciencia ficción y trabajos de investigación como metodologías de aprendizaje de astronomía. El uso del cine de ciencia ficción es significativamente mejor valorado por el alumnado de Navalcarnero que por los alumnos de núcleos urbanos, dándose el caso contrario en la propuesta de trabajos de investigación.

El análisis en función de si los alumnos pertenecen o no a una sección bilingüe muestra más diferencias significativas, en todos los casos con niveles de confianza mayores del 99\%.

De nuevo reaparecen las dos propuestas metodológicas mencionadas en el análisis previo: los trabajos de investigación son significativamente mejor valorados por los alumnos de la sección bilingüe, al contrario de lo que ocurre con la propuesta del cine de ciencia ficción, siendo pequeña la magnitud del efecto asociado en ambos casos.

Las exposiciones orales individuales también son significativamente mejor aceptadas por los alumnos de la sección bilingüe, con un tamaño del efecto asociado moderado. 
JOSÉ LUIS GARCÍA HERRERO

CONOCIMIENTOS ASTRONÓMICOS DEL PROFESORADO DE EDUCACIÓN SECUNDARIA OBLIGATORIA Y PREFERENCIAS METODOLÓGICAS PARA LA ENSEÑANZA DE ASTRONOMÍA

\begin{tabular}{|c|c|c|c|c|c|c|c|}
\hline \multicolumn{8}{|c|}{ ALUMNOS EN FUNCIÓN DE BILINGÜISMO } \\
\hline \multirow{3}{*}{$\begin{array}{l}\text { Metodologías para } \\
\text { el aprendizaje de } \\
\text { astronomía }\end{array}$} & \multirow{2}{*}{\multicolumn{2}{|c|}{$\begin{array}{l}\text { Test de } \\
\text { Levene }\end{array}$}} & \multirow{2}{*}{\multicolumn{3}{|c|}{$\begin{array}{c}\text { Test de } \\
\text { Mann-Whitney }\end{array}$}} & \multirow{2}{*}{\multicolumn{2}{|c|}{$\frac{\text { Media }}{\text { Sección bilingüe }}$}} \\
\hline & & & & & & & \\
\hline & $\mathrm{F}$ & $\mathrm{p}$ & U & $\mathrm{p}$ & $\mathrm{r}$ & No & Sí \\
\hline $\begin{array}{l}\text { Trabajos de } \\
\text { investigación }\end{array}$ & 8,281 & 0,004 & 3872,0 & 0,007 & 0,162 & 3,684 & 4,154 \\
\hline \multirow{3}{*}{$\begin{array}{c}\text { Metodologías para } \\
\text { el aprendizaje de } \\
\text { astronomía }\end{array}$} & \multirow{2}{*}{\multicolumn{5}{|c|}{ Análisis de varianza }} & \multicolumn{2}{|c|}{ Media } \\
\hline & & & & & & \multicolumn{2}{|c|}{ Sección bilingüe } \\
\hline & \multicolumn{2}{|c|}{$\mathrm{F}$} & \multicolumn{2}{|l|}{$\mathrm{p}$} & $\mathrm{d}$ & No & Sí \\
\hline $\begin{array}{l}\text { Exposiciones orales } \\
\text { en parejas o en } \\
\text { grupos }\end{array}$ & \multicolumn{2}{|c|}{13,752} & \multicolumn{2}{|c|}{0,000} & 0,446 & 2,871 & 3,591 \\
\hline $\begin{array}{l}\text { Exposiciones orales } \\
\text { individuales }\end{array}$ & \multicolumn{2}{|c|}{9,805} & \multicolumn{2}{|c|}{0,002} & 0,376 & 2,588 & 3,182 \\
\hline $\begin{array}{l}\text { Proyección de } \\
\text { películas de ciencia } \\
\text { ficción }\end{array}$ & \multicolumn{2}{|c|}{9,165} & \multicolumn{2}{|c|}{0,003} & 0,364 & 3,178 & 2,523 \\
\hline
\end{tabular}

En el caso de las exposiciones orales en parejas o grupos, reaparece una mejor valoración estadísticamente significativa por parte de los alumnos de la sección bilingüe, y en este caso la magnitud del efecto es media.

Por último, se ofrecen los resultados arrojados por los contrastes de medias asociados a profesores y alumnos:

\begin{tabular}{|l|c|c|c|c|c|c|c|c|}
\hline \multicolumn{7}{|c|}{ ANÁLISIS EN FUNCIÓN DEL ESTATUS EDUCATIVO (PROFESOR-ALUMNO) } \\
\hline \multirow{2}{*}{$\begin{array}{c}\text { Metodologías para el } \\
\text { aprendizaje de astronomía }\end{array}$} & \multicolumn{2}{|c|}{ Test de Levene } & \multicolumn{2}{c|}{ Test de Mann-Whitney } & \multicolumn{3}{c|}{ Media } \\
\cline { 2 - 9 } & $\mathrm{F}$ & $\mathrm{p}$ & $\mathrm{U}$ & $\mathrm{p}$ & $\mathrm{r}$ & Profesor & Alumno \\
\hline $\begin{array}{l}\text { Exposiciones orales } \\
\text { individuales }\end{array}$ & 21,713 & 0,000 & 2867,5 & 0,000 & 0,376 & 3,900 & 2,682 \\
\hline $\begin{array}{l}\text { Exposiciones orales en } \\
\text { parejas o en grupos }\end{array}$ & 11,072 & 0,001 & 3599,5 & 0,000 & 0,307 & 4,000 & 2,985 \\
\hline Trabajos de investigación & 12,085 & 0,001 & 3699,5 & 0,000 & 0,305 & 4,560 & 3,758 \\
\hline $\begin{array}{l}\text { Elaboración de trabajos } \\
\text { manuales como maquetas, } \\
\text { relojes de sol, calendarios... }\end{array}$ & 16,096 & 0,000 & 4015,0 & 0,000 & 0,263 & 4,306 & 3,518 \\
\hline $\begin{array}{l}\text { Trabajos escritos } \\
\text { individuales }\end{array}$ & 5,822 & 0,016 & 4282,0 & 0,000 & 0,247 & 3,280 & 2,599 \\
\hline $\begin{array}{l}\text { Observación del cielo con } \\
\text { telescopio o prismáticos }\end{array}$ & 18,509 & 0,000 & 4608,5 & 0,000 & 0,228 & 4,740 & 4,185 \\
\hline $\begin{array}{l}\text { Conferencias de astrónomos } \\
\text { profesionales }\end{array}$ & 15,108 & 0,000 & 4763,0 & 0,000 & 0,203 & 4,300 & 3,673 \\
\hline
\end{tabular}


JOSÉ LUIS GARCÍA HERRERO

CONOCIMIENTOS ASTRONÓMICOS DEL PROFESORADO DE EDUCACIÓN SECUNDARIA OBLIGATORIA Y PREFERENCIAS METODOLÓGICAS PARA LA ENSEÑANZA DE ASTRONOMÍA

\begin{tabular}{|c|c|c|c|c|c|c|c|}
\hline \multicolumn{8}{|c|}{ ANÁLISIS EN FUNCIÓN DEL ESTATUS EDUCATIVO (PROFESOR-ALUMNO) } \\
\hline \multirow{2}{*}{$\begin{array}{l}\text { Metodologías para el } \\
\text { aprendizaje de astronomía }\end{array}$} & \multicolumn{2}{|c|}{ Test de Levene } & \multicolumn{3}{|c|}{ Test de Mann-Whitney } & \multicolumn{2}{|c|}{ Media } \\
\hline & $\mathrm{F}$ & $\mathrm{p}$ & $\mathrm{U}$ & $\mathrm{p}$ & $\mathrm{r}$ & Profesor & Alumno \\
\hline Visitas al planetario & 9,081 & 0,003 & 5490,5 & 0,018 & 0,131 & 4,633 & 4,291 \\
\hline Proyección de documentales & 4,333 & 0,038 & 5773,0 & 0,047 & 0,110 & 4,280 & 3,936 \\
\hline \multirow{2}{*}{$\begin{array}{l}\text { Metodologías para el } \\
\text { aprendizaje de astronomía }\end{array}$} & \multicolumn{5}{|c|}{ Análisis de varianza } & \multicolumn{2}{|c|}{ Media } \\
\hline & \multicolumn{2}{|c|}{$\mathrm{F}$} & $\mathrm{p}$ & & $\mathrm{D}$ & Profesor & Alumno \\
\hline $\begin{array}{l}\text { Uso de noticias de la prensa } \\
\text { escrita }\end{array}$ & \multicolumn{2}{|c|}{70,893} & 0,000 & \multicolumn{2}{|c|}{0,934} & 4,360 & 3,083 \\
\hline $\begin{array}{l}\text { Uso de programas } \\
\text { informáticos sobre } \\
\text { astronomía }\end{array}$ & \multicolumn{2}{|c|}{21,044} & 0,000 & \multicolumn{2}{|c|}{0,508} & 4,438 & 3,733 \\
\hline $\begin{array}{l}\text { Trabajos escritos en parejas } \\
\text { o en grupos }\end{array}$ & \multicolumn{2}{|c|}{6,804} & 0,010 & \multicolumn{2}{|c|}{0,289} & 3,520 & 3,086 \\
\hline
\end{tabular}

En este caso, las diferencias estadísticamente significativas abundan. Como se puede constatar en la tabla anterior, fue necesario aplicar el test de MannWhitney en la mayoría de los casos, debido a la conjunción de una diferencia considerable entre los tamaños de las muestras y la ausencia de homogeneidad de sus varianzas.

De las 15 propuestas metodológicas contenidas en el cuestionario, el análisis arrojó diferencias estadísticamente significativas entre las valoraciones de profesores y alumnos para 12 propuestas. En todos los casos, las diferencias significativas se dieron a favor del profesorado, siendo en 9 ocasiones el nivel de confianza mayor del 99,9\%. Los resultados se muestran, para cada prueba, en orden descendente de la magnitud del tamaño del efecto asociado.

La propuesta metodológica que presenta mayor tamaño del efecto es el uso en el aula de noticias publicadas en la prensa escrita, con un valor cercano a la unidad, denotando un tamaño del efecto grande.

En segundo lugar se sitúa la propuesta de realizar exposiciones orales individuales, con un tamaño del efecto de magnitud muy considerable.

Las exposiciones orales en pareja o grupo, la realización de trabajos de investigación y el uso de programas informáticos presentan una magnitud media en el tamaño del efecto.

En el caso de la confección de trabajos manuales, las observaciones del cielo con telescopio o prismáticos, los trabajos escritos, tanto individuales como en pareja o grupo, y la asistencia a conferencias de astrónomos profesionales, la magnitud del tamaño del efecto asociado es moderada.

Por último, las visitas al planetario y la proyección de documentales son las propuestas metodológicas que presentan magnitudes pequeñas del tamaño del efecto, siempre teniendo en cuenta que se trata de propuestas con diferencias estadísticamente significativas. 


\section{CONCLUSIONES}

Con la debida cautela, teniendo en cuenta el tamaño de la muestra disponible, los resultados sobre conocimientos astronómicos permiten afirmar que el profesorado de ciencias presenta conocimientos afianzados en general sobre astronomía, aunque sería de esperar un nivel de dominio aún mayor de la materia, especialmente en aspectos como la forma de la órbita terrestre, la dinámica de los eclipses solares, el desplazamiento aparente de la bóveda celeste y la relevancia de las constelaciones del zodíaco. Las diferencias son sustanciales al comparar con el profesorado de asignaturas no científicas, ya que en este caso sí se detecta un nivel bajo de conocimientos básicos, superior al nivel del alumnado pero sin una correlación marcada entre sus resultados. De todos modos, hay que tener en cuenta que, en función de su formación, una proporción considerable del profesorado no ha estudiado contenidos sobre astronomía tras finalizar su educación secundaria, y por tanto gran parte su formación al respecto suele presentar un origen autodidacta. Probablemente sería deseable algún tipo de formación específica al respecto, con el fin de paliar las carencias detectadas, especialmente para el profesorado de ciencias. Seguramente sería muy positivo poder realizar un estudio similar con una muestra mucho más representativa, con el fin de que las autoridades educativas pudieran conocer con precisión las necesidades de formación en este campo. De igual manera, sería muy interesante conocer las opiniones de los docentes con relación a qué conceptos consideran importantes para incluir en el currículo, y así decidir si seguir impartiendo conceptos básicos y tradicionales u otros más novedosos y actuales.

Con relación a los mejores resultados obtenidos por los varones en el caso del profesorado, aunque las muestras estaban equilibradas, sería deseable disponer de muestras mayores para poder corroborar tal tendencia en futuros estudios.

En el apartado de las preferencias metodológicas para la enseñanza-aprendizaje de astronomía, podemos concluir que profesores y alumnos consideran importante adoptar enfoques metodológicos innovadores de carácter práctico, esencialmente visitas al planetario, observaciones nocturnas, trabajos de investigación y uso de programas informáticos relacionados con la astronomía.

Las preferencias metodológicas del profesorado se muestran monolíticas, sin variaciones significativas en función de las variables estudiadas. Los resultados arrojados por las opiniones de los alumnos son algo más heterogéneos, con algunas diferencias palpables en función del tipo de población y el nivel académico del alumnado, si tenemos en cuenta que normalmente los grupos de secciones bilingües están formados por los alumnos más brillantes.

Aunque las preferencias de profesores y alumnos coinciden en los aspectos más fundamentales, se detectaron diferencias muy significativas en el grado de valoración de las mismas, lo que parece indicar que el entusiasmo del profesorado por las metodologías propuestas es superior al de los alumnos. 
CONOCIMIENTOS ASTRONÓMICOS DEL PROFESORADO DE EDUCACIÓN SECUNDARIA OBLIGATORIA Y PREFERENCIAS METODOLÓGICAS PARA LA ENSEÑANZA DE ASTRONOMÍA

\section{REFERENCIAS BIBLIOGRÁFICAS}

Abimbola, I. O. (1988). The Problem of Terminology in the Study of Student Conceptions in Science. Science Education, vol. 72, Issue 2, 175-184. http://dx.doi.org/10.1002 sce.3730720206

Bailey, J. M. y Slater, T. F. (2004). A Review of Astronomy Education Research. Astronomy Education Review, vol. 2, Issue 2, 20-45.

Barnett, M. y Morran, J. (2002). Addressing Children's Alternative Frameworks of the Moon's Phases and Eclipses. International Journal of Science Education, vol. 24, Issue 8, 859-879. http://dx.doi.org/10.1080/09500690110095270

Baxter, J. (1989). Children's Understanding of Familiar Astronomical Events. International Journal of Science Education, vol. 11, Issue 5, 502-513. http://dx.doi. org $/ 10.1080 / 0950069890110503$

Beare, R. (2007). Investigation into the potential of investigative projects involving powerful robotic telescopes to inspire interest in science. International Journal of Science Education, vol. 29, n. , 279-306.

Brandou, B. (1997). Backyard Astronomy: Observing Moon Phases. Science and Children, vol. 34, Issue $8,18-21$.

Cohen, J. (1988). Statistical Power Analysis for the Behavioral Sciences. New Jersey: Lawrence Erlbaum Associates. http://dx.doi.org/10.1002/bs.3830330104

Comunidad de Madrid. Consejería de Educación (2007a). Decreto 22/2007, de 10 de mayo, del Consejo de Gobierno, por el que se establece para la Comunidad de Madrid el currículo de la Educación Primaria. BOCM, 29 de mayo de 2007.

- (2007b). Decreto 23/2007, de 10 de mayo, del Consejo de Gobierno, por el que se establece para la Comunidad de Madrid el currículo de la Educación Secundaria Obligatoria. BOCM, 29 de mayo de 2007.

Comunidad de Madrid (2013). Datos y Cifras de la Educación 2012-2013. Madrid: Dirección General de Mejora de la Calidad de la Enseñanza.

Danaia, L. y McKinnon, D. H. (2008). Common Alternative Astronomical Conceptions Encountered in Junior Secondary Science Classes: Why is This So? Astronomy Education Review, vol. 6, Issue 2, 32-53.

Dunlop, J. (2000). How Children Observe the Universe. Publications of the Astronomical Society of Australia, vol. 17, 194-206. http://dx.doi.org/10.1071/AS00194

Field, A. (2009). Discovering statistics using SPSS. London: SAGE.

Foster, G. W. (1996). Look to the Moon. Science and Children, 34 (3), 30-33.

Gilbert, J. K. y Swift, D. J. (1985). Towards a Lakatosian Analysis of the Piagetian and Alternative Conceptions Research Programs. Science Education, vol. 69, Issue 5, 681-696. http://dx.doi.org/10.1002/sce.3730690510

Hair, J. F.; Black, W. C.; Babin, B. J.; Anderson, R. E. y Tatham, R. L. (2006). Multivariate data analysis. New Jersey: Pearson Prentice Hall.

Hufnagel, B. (2002). Development of the Astronomy Diagnostic Test. Astronomy Education Review, vol. 1, Issue 1, 47-51.

Instituto Nacional de Estadística (2013). Cifras de población resultantes de la Revisión del Padrón municipal a 1 de enero de 2012. http://www.ine.es/INEBASE/temas/t20/e260/ a2012/10/pobmun12.xls. Descargado el 23-7-2013.

Jefatura del Estado (2006). Ley Orgánica de Educación. BOE, 4 de mayo de 2006. 
CONOCIMIENTOS ASTRONÓMICOS DEL PROFESORADO DE EDUCACIÓN SECUNDARIA OBLIGATORIA Y PREFERENCIAS METODOLÓGICAS PARA LA ENSEÑANZA DE ASTRONOMÍA

Jones, B. (1988). Primary teacher students' explanations of the day and night, the seasons and crescent Moon. Conference of the New Zealand Association for Research in Education. Massey University, Palmerston North, New Zealand.

Jones, B. L.; Lynch, P. P. y Reesink, C. (1987). Children's Conceptions of the Earth, Sun and Moon. International Journal of Science Education, vol. 9, Issue 1, 43-53. http://dx.doi. org/10.1080/0950069870090106

Kanssagalo, M. (1994). Children's independent exploration of a natural phenomenon by using a pictorial computer-based simulation. Journal of Computing in Childhood Education, 5, 285-297.

Kerlinger, F. N. (1979). Foundations of Behavioural Research. New York: Holt Rinehart \& Winston.

Kline, P. (1999). The handbook of psychological testing. London: Routledge.

Marshall, L. A. (1996). Bringing the Moon into the classroom. The Physics Teacher, n. ${ }^{\circ} 34,360-361$. http://dx.doi.org/10.1119/1.2344481

Martínez, B.; Pérez, P. y Boix, A. (2003). Observaciones y modelos en astronomía. Descargado el 17-7-2013. http://recursostic.educacion.es/apls/informacion_didactica/679.

Ministerio de Educación y Ciencia (Arribas y Granados, 1992). OPTATIVAS: Taller de Astronomía. Madrid: Secretaría General Técnica. Centro de Publicaciones.

Morales, P. (2008). Estadística Aplicada a las Ciencias Sociales. Madrid: Universidad Pontificia Comillas.

- (2010). Análisis de ítems en pruebas objetivas. Descargado el 8-9-2012. http://www. upcomillas.es/personal/peter/principal.htm.

Pasachoff, J. M. (2002). What Should College Students Learn? Astronomy Education Review, vol. 1, Issue 1, 124-130.

Pérez, R.; García, J. L.; Gil, J. A. y Galán, A. (2009). Estadística aplicada a la Educación. Madrid: Pearson-UNED.

Pérez, U.; Álvarez, M. ${ }^{\mathrm{a}}$ y Prieto, J. (2008). The Historical Evolution of Knowledge of the Universe: Errors in Secondary Education Textbooks in Spain. Astronomy Education Review, vol. 7, Issue 1, 23-26. http://dx.doi.org/10.3847/AER2008003

Sadler, P. (2001). Choosing Between Teaching Helioseismology and Phases of the Moon. The Physics Teacher, n.. 39, 554-555. http://dx.doi.org/10.1119/1.1482566

Schneps, M. (1989). A Private Universe (archivo de video - 20:49 min.) San Francisco: Astronomical Society of the Pacific. Descargado el 22-1-2010. http://www.learner.org/ resources/series28.html?pop= yes\&pid=9\#.

Sevillano García, M. ${ }^{a}$ L. (1998). Estrategias Innovadoras para una Enseñanza de Calidad. Madrid: Pearson.

- (2008). Estrategias de Enseñanza y Aprendizaje. Madrid: UNED.

Thorndike, R. M. (1997). Measurement and evaluation in psychology and education. New York: MacMillan.

Trumper, R. (2000). University Students' Conceptions of Basic Astronomy Concepts. Physics Education, vol. 35, Issue 1, 9-15. http://dx.doi.org/10.1088/0031-9120/35/1/301

- (2001). A Cross-Age Study of Junior High School Students' Conceptions of Basic Astronomy Concepts. International Journal of Science Education, vol. 23, Issue 11, 1111-1123.

Vega, A. (2001). Tenerife tiene seguro de Sol (y de Luna). Representaciones del profesorado acerca del día y la noche. Enseñanza de las Ciencias, vol. 19, 1, 31-44.

- (2007). Ideas, conocimientos y teorías de niños y adultos sobre las relaciones Sol-TierraLuna. Estado actual de las investigaciones. Revista de Educación, 342, 475-500, 2007.

Zeilik, M. (2003). Birth of the Astronomy Diagnostic Test: Prototest Evolution. Astronomy Education Review, vol. 2, Issue 1, 46-52. 
CONOCIMIENTOS ASTRONÓMICOS DEL PROFESORADO DE EDUCACIÓN SECUNDARIA OBLIGATORIA Y PREFERENCIAS METODOLÓGICAS PARA LA ENSEÑANZA DE ASTRONOMÍA

ANEXO I. CUESTIONARIO SOBRE TIC Y ASTRONOMÍA PARA PROFESORES

Por medio de este cuestionario anónimo se pretende estudiar las actitudes de los profesores de ciencias hacia las Tecnologías de la Información y Comunicación (TIC) y sus conocimientos sobre astronomía.

1.1) Usted es...

1.2) Imparte clase en...
- A: Varón

- A: Secundaria
- B: Mujer

- B: Primaria

1.3) Pertenece a la/s especialidad/es de:

2) Uso las Tic... (marque una respuesta por fila)

\begin{tabular}{|l|c|c|c|c|}
\hline & $\begin{array}{c}\text { NO LAS } \\
\text { UTILIZO }\end{array}$ & OCASIONALMENTE & $\begin{array}{c}\text { CASI } \\
\text { SIEMPRE }\end{array}$ & SIEMPRE \\
\hline $\begin{array}{l}\text { 1. Como apoyo a la exposición oral de } \\
\text { contenidos }\end{array}$ & 1 & 2 & 3 & 4 \\
\hline $\begin{array}{l}\text { 2. Para comunicarme con mis alumnos } \\
\text { en la resolución de dudas y consultas }\end{array}$ & 1 & 2 & 3 & 4 \\
\hline $\begin{array}{l}\text { 3. Para monitorizar directamente la } \\
\text { evolución del proceso de aprendizaje } \\
\text { de mis alumnos }\end{array}$ & 1 & 2 & 3 & 4 \\
\hline $\begin{array}{l}\text { 4. Para elaborar materiales didácticos } \\
\text { que uso en clase }\end{array}$ & 1 & 2 & 3 & 4 \\
\hline $\begin{array}{l}\text { 5. Para introduciry mantener contenidos } \\
\text { propios de mi asignatura a una página } \\
\text { web, blog o similar a los que tienen } \\
\text { acceso mis alumnos }\end{array}$ & 1 & 2 & 3 & 4 \\
\hline $\begin{array}{l}\text { 6. Para mantener un registro de los } \\
\text { datos que utilizo para la evaluación de } \\
\text { los alumnos }\end{array}$ & 1 & 2 & 3 & 4 \\
\hline $\begin{array}{l}\text { 7. Para comunicarme con las familias de } \\
\text { mis alumnos e intercambiar información } \\
\text { acerca de su proceso educativo }\end{array}$ & 1 & 2 & 3 & 4 \\
\hline $\begin{array}{l}\text { 8. Para trabajar y colaborar con mis } \\
\text { compañeros/as de ciclo, nivel o } \\
\text { departamento en tareas relacionadas } \\
\text { con mis actividades docentes }\end{array}$ & 1 & 2 & 3 & 4 \\
\hline
\end{tabular}

Ahora pasamos a las preguntas sobre astronomía

Debe marcar una respuesta para cada pregunta (a no ser que se indique otra cosa). Si no sabe qué responder a alguna pregunta, no es necesario que conteste 
CONOCIMIENTOS ASTRONÓMICOS DEL PROFESORADO DE EDUCACIÓN SECUNDARIA OBLIGATORIA Y PREFERENCIAS METODOLÓGICAS PARA LA ENSEÑANZA DE ASTRONOMÍA

3.1) ¿Hay algún día del año en que la duración del día sea igual a la de la noche?
- A: Ninguno
- B: Uno
- C: Dos
- D: Más de dos

3.2) En España, si un día tiene 14 horas de luz, ¿en qué estación (o estaciones) nos encontramos?
- A: Primavera
- B: Verano
- C: Otoño
- D: Invierno

3.3) ¿Por qué existen las estaciones del año?

- A: Por el giro de la Luna alrededor de la Tierra

- B: Por la inclinación del eje de rotación de la Tierra

- C: Por el movimiento del Sol alrededor de la Tierra

- D: Por el cambio en la distancia de la Tierra al Sol

3.4) Si observa la Luna llena salir por el Este; ¿qué hora del día cree que puede ser?
- A: Amanecer
- B: Atardecer
- C: Mediodía
- D: Medianoche

3.5) ¿Cuál de los siguientes dibujos muestra con mayor exactitud la órbita de la Tierra alrededor del Sol?
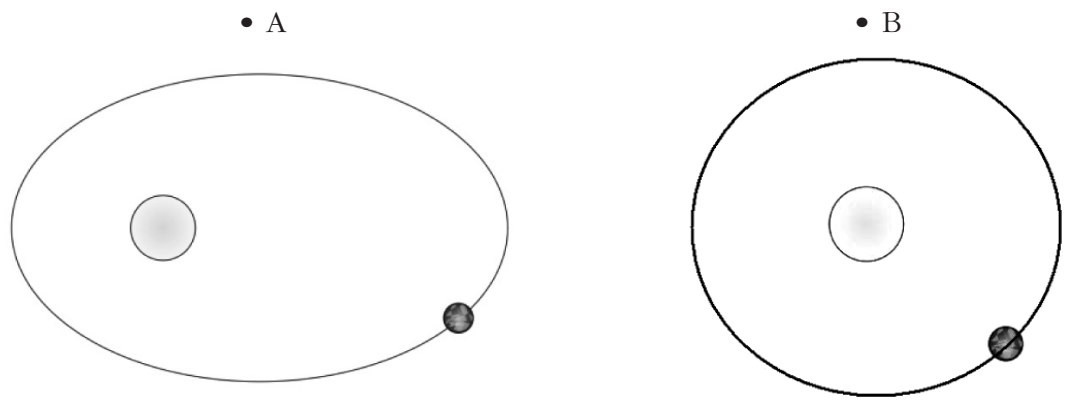

3.6) ¿En Madrid, cuántas veces al año está el Sol directamente sobre nuestra cabeza (es decir, sin producir sombra)?

- A: Todos los días al mediodía

- B: Solamente el primer día del verano

- C: Solamente el primer día del invierno

- D: El primer día de la primavera y del otoño

- E: Ninguno

3.7) ¿Por qué cree que hace más calor en verano?

- A: Porque la Tierra está más cerca del Sol

- B: Porque el eje de la Tierra está inclinado hacia el Sol 
CONOCIMIENTOS ASTRONÓMICOS DEL PROFESORADO DE EDUCACIÓN SECUNDARIA OBLIGATORIA Y PREFERENCIAS METODOLÓGICAS PARA LA ENSEÑANZA DE ASTRONOMÍA

- C: Porque en verano hay menos nubes y lluvias

- D: Porque hay más horas de luz

3.8) Las siguientes afirmaciones se refieren a la estrella polar. Indique si cree que son verdaderas $(\mathrm{V})$ o falsas $(\mathrm{F})$

- A: Es parte de la Osa Mayor $\Rightarrow$

- B: Se encuentra en el punto más alto del cielo $\Rightarrow$

- C: Se ve desde cualquier parte de la Tierra $\Rightarrow$

- D: Siempre indica el Norte $\Rightarrow$

3.9) El jefe indio ha dicho: "Mi hija cumple hoy diez lunas". ¿Qué edad tiene su hija?
- A: 10 días
- B: 10 semanas
- C: 10 meses
- D: 10 años

3.10) ¿En qué fase debe encontrarse la Luna para que se pueda producir un eclipse de Sol?

- A: Luna nueva

- B: Luna llena

- C: Cuarto menguante

- D: Cuarto creciente

3.11) ¿Por qué siempre vemos la misma cara de la Luna?

- A: Porque la Luna no tiene movimiento de rotación sobre su eje

- B: Porque coinciden el período de rotación y el de traslación

- C: No es cierto, desde la Tierra vemos la superficie completa de la Luna

- D: Porque la Luna no se traslada alrededor de la Tierra

3.12) ¿Qué tiene que ocurrir para que se produzca un eclipse de Luna?

- A: Que el Sol se sitúe entre la Tierra y la Luna

- B: Que la Luna se sitúe entre el Sol y la Tierra

- C: Que la Tierra se sitúe entre el Sol y la Luna

- D: Que el Sol y la Luna estén a la misma distancia de la Tierra

3.13) ¿Cómo se denomina la franja del cielo por la que se desplazan el Sol, la Luna y los planetas en el cielo?
- A: Meridiano
- B: Vía Láctea
- C: Eclíptica
- D: Camino de Santiago

3.14) ¿Cuál es la importancia de las constelaciones del zodíaco?

- A: Determinan el destino de las personas

- B: Contienen a las estrellas más cercanas a la Tierra

- C: Los planetas se mueven a través de ellas

- D: Son las más grandes del cielo 
CONOCIMIENTOS ASTRONÓMICOS DEL PROFESORADO DE EDUCACIÓN SECUNDARIA OBLIGATORIA Y PREFERENCIAS METODOLÓGICAS PARA LA ENSEÑANZA DE ASTRONOMÍA

3.15) Así se vería el cielo al mediodía en cierta fecha si pudiéramos ver las estrellas durante el día: el Sol se encuentra entre las estrellas de la constelación de Géminis. ¿Cerca de qué constelación esperarías localizar al Sol al atardecer?
- A: Leo
- B: Cáncer
- C: Géminis
- D: Tauro
- E: Piscis

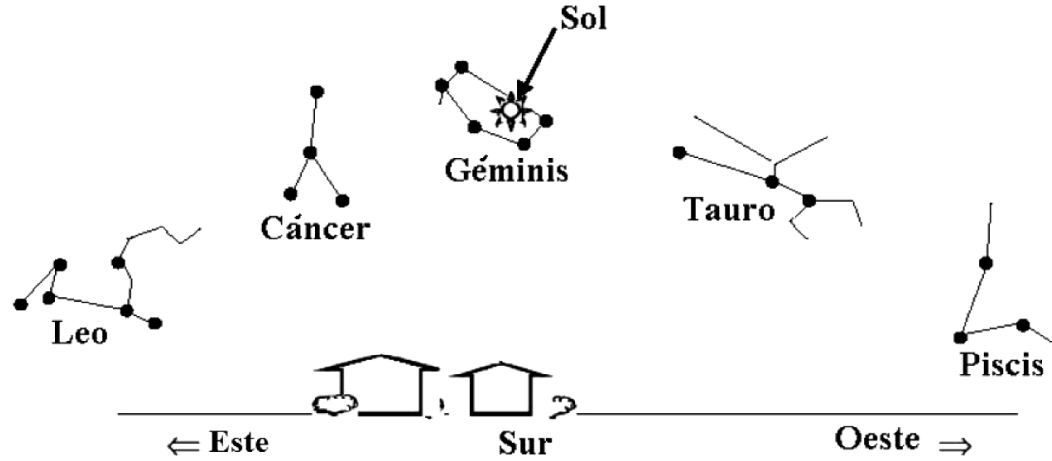

3.16) Los movimientos del Sol son:

- A: Rotación alrededor de su eje

- B: El Sol se encuentra totalmente en reposo

- C: Traslación alrededor de la Tierra

- D: Rotación alrededor de su eje y translación alrededor del centro de la Vía Láctea

3.17) El siguiente diagrama muestra la Tierra, el Sol y 5 posibles posiciones diferentes para la Luna. ¿Qué posición de la Luna causaría que se observarse desde la Tierra como en la figura de la derecha?
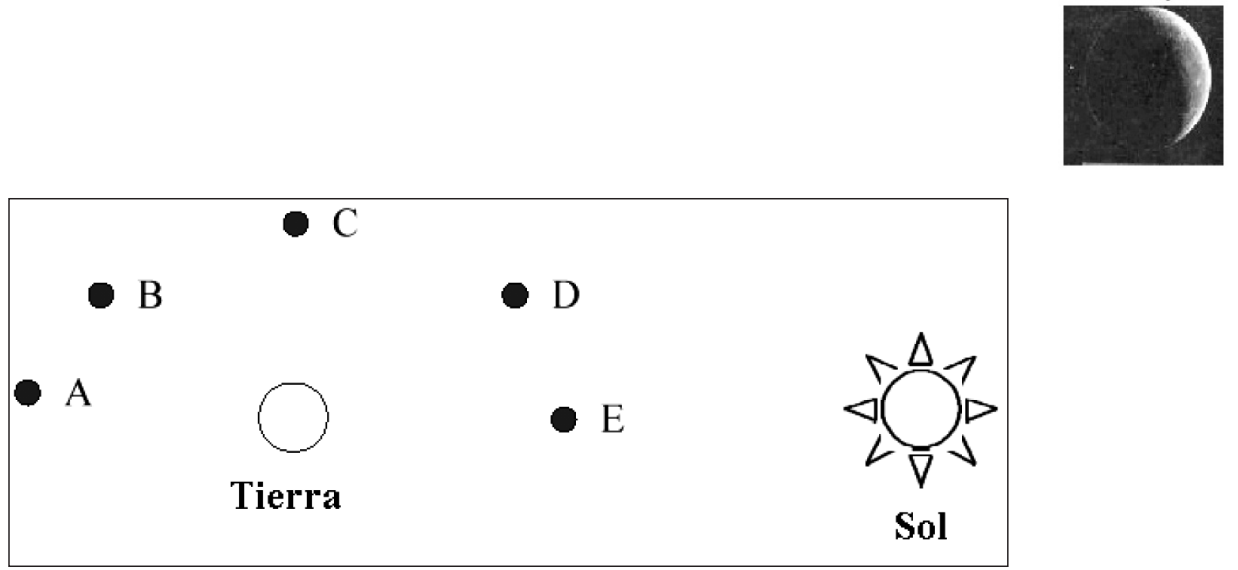
CONOCIMIENTOS ASTRONÓMICOS DEL PROFESORADO DE EDUCACIÓN SECUNDARIA OBLIGATORIA Y PREFERENCIAS METODOLÓGICAS PARA LA ENSEÑANZA DE ASTRONOMÍA

3.18) Identifique la constelación de Orión:

- A

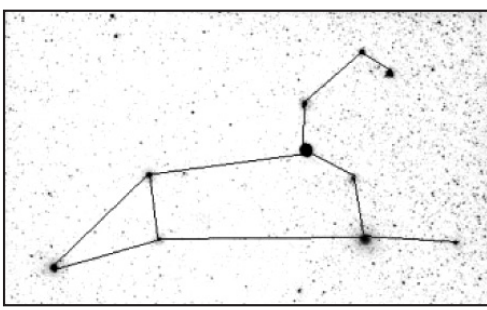

- $\mathrm{C}$

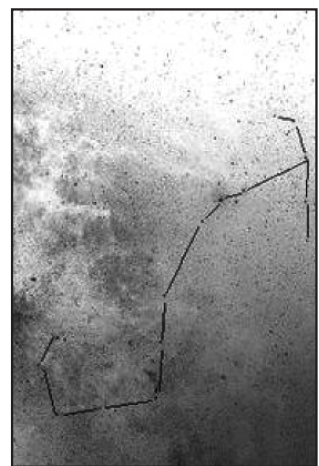

- B

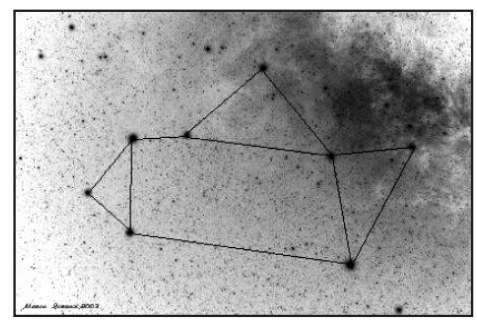

- D

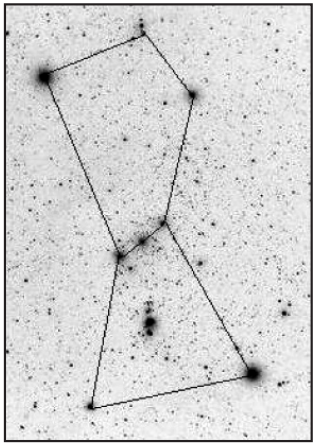

3.19) ¿Cuál es el estado actual del Universo?

- A: Se está expandiendo, aunque los objetos cercanos se atraen

- B: Se está contrayendo.

- C: Está en estado de reposo, ni se expande ni se contrae 
JOSÉ LUIS GARCÍA HERRERO

CONOCIMIENTOS ASTRONÓMICOS DEL PROFESORADO DE EDUCACIÓN SECUNDARIA OBLIGATORIA Y PREFERENCIAS METODOLÓGICAS PARA LA ENSEÑANZA DE ASTRONOMÍA

4) Valore cada opción con el código correspondiente, según su idoneidad para la enseñanza de la astronomía.

\begin{tabular}{|c|c|c|c|c|c|}
\hline & $\begin{array}{c}\text { MUY EN } \\
\text { DESACUERDO }\end{array}$ & $\begin{array}{l}\text { BASTANTE EN } \\
\text { DESACUERDO }\end{array}$ & $\begin{array}{c}\text { NI } \\
\text { ACUERDO/NI } \\
\text { DESACUERDO }\end{array}$ & $\begin{array}{l}\text { BASTANTE } \\
\text { DE } \\
\text { ACUERDO } \\
\end{array}$ & $\begin{array}{l}\text { MUY DE } \\
\text { ACUERDO }\end{array}$ \\
\hline $\begin{array}{l}\text { 1. Explicación } \\
\text { tradicional del profesor }\end{array}$ & 1 & 2 & 3 & 4 & 5 \\
\hline $\begin{array}{l}\text { 2. Trabajos escritos } \\
\text { individuales }\end{array}$ & 1 & 2 & 3 & 4 & 5 \\
\hline $\begin{array}{l}\text { 3. Trabajos escritos en } \\
\text { parejas o en grupos }\end{array}$ & 1 & 2 & 3 & 4 & 5 \\
\hline 4. Debates & 1 & 2 & 3 & 4 & 5 \\
\hline $\begin{array}{l}\text { 5. Proyección de } \\
\text { documentales }\end{array}$ & 1 & 2 & 3 & 4 & 5 \\
\hline $\begin{array}{l}\text { 6. Proyección de } \\
\text { películas de ciencia } \\
\text { ficción }\end{array}$ & 1 & 2 & 3 & 4 & 5 \\
\hline $\begin{array}{l}\text { 7. Uso de programas } \\
\text { informáticos sobre } \\
\text { astronomía }\end{array}$ & 1 & 2 & 3 & 4 & 5 \\
\hline $\begin{array}{l}\text { 8. Uso de noticias } \\
\text { publicadas en la prensa } \\
\text { escrita }\end{array}$ & 1 & 2 & 3 & 4 & 5 \\
\hline $\begin{array}{l}\text { 9. Exposiciones orales } \\
\text { individuales }\end{array}$ & 1 & 2 & 3 & 4 & 5 \\
\hline $\begin{array}{l}\text { 10. Exposiciones orales } \\
\text { en parejas o en grupos }\end{array}$ & 1 & 2 & 3 & 4 & 5 \\
\hline $\begin{array}{l}\text { 11. Observación del } \\
\text { cielo con telescopio o } \\
\text { prismáticos }\end{array}$ & 1 & 2 & 3 & 4 & 5 \\
\hline 12. Visitas al planetario & 1 & 2 & 3 & 4 & 5 \\
\hline $\begin{array}{l}\text { 13. Trabajos de } \\
\text { investigación }\end{array}$ & 1 & 2 & 3 & 4 & 5 \\
\hline $\begin{array}{l}\text { 14. Conferencias } \\
\text { de astrónomos } \\
\text { profesionales }\end{array}$ & 1 & 2 & 3 & 4 & 5 \\
\hline $\begin{array}{l}\text { 15. Elaboración de } \\
\text { trabajos manuales como } \\
\text { maquetas, relojes de sol, } \\
\text { calendarios... }\end{array}$ & 1 & 2 & 3 & 4 & 5 \\
\hline
\end{tabular}


JOSÉ LUIS GARCÍA HERRERO

CONOCIMIENTOS ASTRONÓMICOS DEL PROFESORADO DE EDUCACIÓN SECUNDARIA OBLIGATORIA Y PREFERENCIAS METODOLÓGICAS PARA LA ENSEÑANZA DE ASTRONOMÍA

Anexo II. Resultados Globales Del CUestionario sobre ASTRONOMía PARA PROFESORES

\begin{tabular}{|c|c|c|c|c|c|c|c|c|}
\hline & 3.1 & 3.2 & 3.2 & 3.3 & 3.4 & 3.5 & 3.6 & 3.7 \\
\hline $\mathrm{A}$ & $0 \%$ & $12 \%$ & $\mathrm{AB}$ & $0 \%$ & $8 \%$ & $82 \%$ & $30 \%$ & $14 \%$ \\
\hline $\mathrm{B}$ & $12 \%$ & $56 \%$ & $20 \%$ & $74 \%$ & $64 \%$ & $14 \%$ & $10 \%$ & $76 \%$ \\
\hline $\mathrm{C}$ & $86 \%$ & $2 \%$ & $\mathrm{AC}$ & $24 \%$ & $2 \%$ & & $0 \%$ & $2 \%$ \\
\hline $\mathrm{D}$ & $0 \%$ & $0 \%$ & $8 \%$ & $0 \%$ & $8 \%$ & & $14 \%$ & $4 \%$ \\
\hline $\mathrm{E}$ & & & $\mathrm{BC}$ & & & & $40 \%$ & \\
\hline $\mathrm{Ns} / \mathrm{Nc}$ & $0 \%$ & $0 \%$ & $2 \%$ & $0 \%$ & $0 \%$ & $0 \%$ & $0 \%$ & $0 \%$ \\
\hline
\end{tabular}

\begin{tabular}{|c|c|c|c|c|c|c|c|c|}
\hline & $3.8 \mathrm{~A}$ & $3.8 \mathrm{~B}$ & $3.8 \mathrm{C}$ & $3.8 \mathrm{D}$ & 3.9 & 3.10 & 3.11 & 3.12 \\
\hline $\mathrm{A}$ & $\mathrm{V}$ & $\mathrm{V}$ & $\mathrm{V}$ & $\mathrm{V}$ & $2 \%$ & $60 \%$ & $18 \%$ & $6 \%$ \\
\hline $\mathrm{B}$ & $20 \%$ & $6 \%$ & $8 \%$ & $86 \%$ & $6 \%$ & $28 \%$ & $78 \%$ & $12 \%$ \\
\hline $\mathrm{C}$ & $\mathrm{F}$ & $\mathrm{F}$ & $\mathrm{F}$ & $\mathrm{F}$ & $92 \%$ & $2 \%$ & $2 \%$ & $78 \%$ \\
\hline $\mathrm{D}$ & $80 \%$ & $94 \%$ & $92 \%$ & $14 \%$ & $0 \%$ & $0 \%$ & $0 \%$ & $0 \%$ \\
\hline $\mathrm{Ns} / \mathrm{NC}$ & & & & & $0 \%$ & $0 \%$ & $0 \%$ & $0 \%$ \\
\hline
\end{tabular}

\begin{tabular}{|c|c|c|c|c|c|c|c|}
\hline & 3.13 & 3.14 & 3.15 & 3.16 & 3.17 & 3.18 & 3.19 \\
\hline $\mathrm{A}$ & $2 \%$ & $2 \%$ & $4 \%$ & $4 \%$ & $12 \%$ & $8 \%$ & $96 \%$ \\
\hline $\mathrm{B}$ & $14 \%$ & $40 \%$ & $8 \%$ & $12 \%$ & $22 \%$ & $8 \%$ & $2 \%$ \\
\hline $\mathrm{C}$ & $78 \%$ & $42 \%$ & $22 \%$ & $0 \%$ & $10 \%$ & $2 \%$ & $2 \%$ \\
\hline $\mathrm{D}$ & $0 \%$ & $2 \%$ & $8 \%$ & $82 \%$ & $42 \%$ & $70 \%$ & \\
\hline $\mathrm{E}$ & & & $52 \%$ & & $4 \%$ & & \\
\hline $\mathrm{Ns} / \mathrm{Nc}$ & $0 \%$ & $0 \%$ & $0 \%$ & $0 \%$ & $0 \%$ & $0 \%$ & $0 \%$ \\
\hline
\end{tabular}


196

JOSÉ LUIS GARCÍA HERRERO

CONOCIMIENTOS ASTRONÓMICOS DEL PROFESORADO DE EDUCACIÓN SECUNDARIA OBLIGATORIA Y PREFERENCIAS METODOLÓGICAS PARA LA ENSEÑANZA DE ASTRONOMÍA

ANEXO III

A continuación se muestran los análisis de fiabilidad completos de los cuestionarios utilizados, obtenidos por medio de spss 17.0.

CUESTIONARIO DE CONCEPTOS ASTRONÓMICOS-PROFESORADO

\begin{tabular}{|c|c|c|c|c|}
\hline \multicolumn{5}{|c|}{ ITEM-TOTAL STATISTICS } \\
\hline C1 & $\begin{array}{c}\text { Scale Mean if Item } \\
\text { Deleted }\end{array}$ & $\begin{array}{c}\text { Scale Variance if } \\
\text { Item Deleted }\end{array}$ & $\begin{array}{c}\text { Corrected Item- } \\
\text { Total Correlation }\end{array}$ & $\begin{array}{c}\text { Cronbach's Alpha } \\
\text { if Item Deleted }\end{array}$ \\
\hline C2 & 13,50 & 12,296 &, 241 &, 765 \\
\hline C3 & 14,16 & 12,953 &,- 036 &, 783 \\
\hline C4 & 13,62 & 11,628 &, 393 &, 755 \\
\hline C5 & 13,72 & 12,410 &, 107 &, 777 \\
\hline C6 & 14,22 & 12,216 &, 274 &, 763 \\
\hline C7 & 13,26 & 12,360 &, 262 &, 764 \\
\hline C8A & 13,56 & 11,306 &, 523 &, 746 \\
\hline C8B & 13,42 & 11,802 &, 376 &, 757 \\
\hline C8C & 13,44 & 13,147 &,- 111 &, 778 \\
\hline C8D & 13,50 & 12,415 &, 270 &, 764 \\
\hline C9 & 13,44 & 12,133 &, 309 &, 761 \\
\hline C10 & 13,76 & 12,619 &, 163 &, 768 \\
\hline C11 & 13,58 & 11,615 &, 341 &, 759 \\
\hline C12 & 13,58 & 11,269 &, 558 &, 744 \\
\hline C13 & 13,58 & 11,473 &, 481 &, 749 \\
\hline C14 & 13,94 & 12,004 &, 287 &, 763 \\
\hline C15 & 14,14 & 11,527 &, 365 &, 758 \\
\hline C16 & 13,54 & 11,307 &, 543 &, 745 \\
\hline C17 & 13,94 & 11,886 &, 364 &, 758 \\
\hline C18 & 13,66 & 11,527 &, 365 &, 758 \\
\hline C19 & 13,40 & 11,249 &, 498 &, 747 \\
\hline
\end{tabular}


JOSÉ LUIS GARCÍA HERRERO

CONOCIMIENTOS ASTRONÓMICOS DEL PROFESORADO DE EDUCACIÓN SECUNDARIA OBLIGATORIA Y PREFERENCIAS METODOLÓGICAS PARA LA ENSEÑANZA DE ASTRONOMÍA

\section{CUESTIONARIO DE CONCEPTOS ASTRONÓMICOS-ALUMNADO}

Este cuestionario está compuesto por un número mayor de preguntas que el del profesorado, pero la parte utilizada para la presente investigación contiene exactamente las mismas preguntas (en fondo gris).

\begin{tabular}{|c|c|c|c|c|}
\hline \multicolumn{5}{|c|}{ ITEM-TOTAL STATISTICS } \\
\hline & \begin{tabular}{|c|} 
Scale Mean if Item \\
Deleted
\end{tabular} & $\begin{array}{l}\text { Scale Variance If } \\
\text { Item Deleted }\end{array}$ & $\begin{array}{l}\text { Corrected Item- } \\
\text { Total Correlation } \\
\end{array}$ & $\begin{array}{l}\text { Cronbach's Alpha } \\
\text { if Item Deleted }\end{array}$ \\
\hline $\mathrm{C} 1$ & \begin{tabular}{|l|}
12,75 \\
\end{tabular} & 14,835 &, 285 &, 594 \\
\hline C-- & 12,38 & 15,196 & 260 & ,599 \\
\hline $\mathrm{C} 2$ & 13,12 & 16,110 &, 000 & ,617 \\
\hline $\mathrm{C} 3$ & 12,73 & 15,217 & ,181 & 605 \\
\hline $\mathrm{C} 4$ & 12,70 & 15,644 & 069 & ,617 \\
\hline $\mathrm{C} 5$ & 13,01 & 16,545 &,- 171 & ,633 \\
\hline C6 & 13,01 & 16,080 &,- 018 &, 622 \\
\hline $\mathrm{C} 7$ & 12,81 & 15,310 &, 166 & ,607 \\
\hline $\mathrm{C} 8 \mathrm{~A}$ & 12,74 & 15,359 & , 144 & 609 \\
\hline $\mathrm{C} 8 \mathrm{~B}$ & 12,63 & 15,252 & , 171 &, 606 \\
\hline $\mathrm{C} 8 \mathrm{C}$ & 12,88 & 15,592 & ,101 & ,613 \\
\hline $\mathrm{C} 8 \mathrm{D}$ & 12,43 & 14,821 &, 343 &, 590 \\
\hline C9 & 12,74 & 15,137 & 203 & 603 \\
\hline C-- & 12,72 & 15,220 &, 180 & ,606 \\
\hline C-- & 12,85 & 15,211 & ,201 & ,603 \\
\hline $\mathrm{C} 10$ & 12,85 & 15,097 & ,232 & 600 \\
\hline C11 & 12,78 & 15,155 & ,202 & ,603 \\
\hline C12 & 12,87 & 14,787 & 330 &, 590 \\
\hline C-- & 12,70 & 15,353 & ,144 & ,609 \\
\hline C13 & 12,87 & 15,001 & ,266 & ,597 \\
\hline C14 & 13,07 & 16,141 &,- 029 &, 620 \\
\hline C15 & 13,10 & 16,011 & 041 & ,615 \\
\hline C-- & 12,55 & 15,434 & ,132 & ,610 \\
\hline C16 & 12,82 & 15,368 & ,152 & ,608 \\
\hline C17 & 12,87 & 15,682 & , 075 & ,616 \\
\hline C18 & 12,79 & 14,874 & , 280 & , 595 \\
\hline C-- & 12,42 & 15,081 & ,269 &, 597 \\
\hline C-- & 12,65 & 15,014 & 233 & ,600 \\
\hline C-- & 13,03 & 16,519 &,- 164 & ,632 \\
\hline C-- & 12,64 & 14,696 & ,319 & ,590 \\
\hline C-- & 12,40 & 15,150 & ,259 & ,599 \\
\hline C19 & 12,60 & 14,706 & 321 & ,590 \\
\hline
\end{tabular}


JOSÉ LUIS GARCÍA HERRERO

CONOCIMIENTOS ASTRONÓMICOS DEL PROFESORADO DE EDUCACIÓN SECUNDARIA OBLIGATORIA Y PREFERENCIAS METODOLÓGICAS PARA LA ENSEÑANZA DE ASTRONOMÍA

CUESTIONARIO DE PREFERENCIAS METODOLÓGICAS-PROFESORADO

\begin{tabular}{|c|c|c|c|c|}
\hline \multicolumn{5}{|c|}{ ITEM-TOTAL STATISTICS } \\
\hline & $\begin{array}{c}\text { Scale Mean if } \\
\text { Item Deleted }\end{array}$ & $\begin{array}{c}\text { Scale Variance if } \\
\text { Item Deleted }\end{array}$ & $\begin{array}{c}\text { Corrected Item- } \\
\text { Total Correlation }\end{array}$ & $\begin{array}{c}\text { Cronbach's Alpha if } \\
\text { Item Deleted }\end{array}$ \\
\hline C1 & 57,4000 & 27,836 &, 275 &, 747 \\
\hline C2 & 57,2222 & 27,949 &, 370 &, 734 \\
\hline C3 & 57,0000 & 26,636 &, 387 &, 733 \\
\hline C4 & 56,5111 & 24,756 &, 664 &, 699 \\
\hline C5 & 56,1333 & 26,755 &, 634 &, 711 \\
\hline C6 & 57,2444 & 28,462 &, 218 &, 754 \\
\hline C7 & 56,0000 & 26,364 &, 531 &, 717 \\
\hline C8 & 56,1111 & 27,465 &, 448 &, 726 \\
\hline C9 & 56,5333 & 28,391 &, 373 &, 734 \\
\hline C10 & 56,4889 & 28,301 &, 297 &, 742 \\
\hline C11 & 55,6889 & 30,265 &, 252 &, 745 \\
\hline C12 & 55,8222 & 29,740 &, 312 &, 741 \\
\hline C13 & 55,8667 & 30,391 &, 193 &, 748 \\
\hline C14 & 56,0889 & 30,856 &, 080 &, 757 \\
\hline C15 & 56,1111 & 29,374 &, 276 &, 743 \\
\hline
\end{tabular}

CUESTIONARIO DE PREFERENCIAS METODOLÓGICAS-ALUMNADO

\begin{tabular}{|c|c|c|c|c|}
\hline \multicolumn{5}{|c|}{ ITEM-TOTAL STATISTICS } \\
\hline & $\begin{array}{c}\text { Scale Mean if } \\
\text { Item Deleted }\end{array}$ & $\begin{array}{c}\text { Scale Variance if } \\
\text { Item Deleted }\end{array}$ & $\begin{array}{c}\text { Corrected Item- } \\
\text { Total Correlation }\end{array}$ & $\begin{array}{c}\text { Cronbach's Alpha } \\
\text { if Item Deleted }\end{array}$ \\
\hline C1 & 48,3378 & 52,505 &, 126 &, 741 \\
\hline C2 & 48,9919 & 50,121 &, 287 &, 726 \\
\hline C3 & 48,5056 & 48,492 &, 380 &, 716 \\
\hline C4 & 47,8515 & 49,741 &, 339 &, 721 \\
\hline C5 & 47,6554 & 50,361 &, 279 &, 727 \\
\hline C6 & 48,5170 & 49,453 &, 226 &, 736 \\
\hline C7 & 47,8629 & 49,785 &, 332 &, 721 \\
\hline C8 & 48,5081 & 48,775 &, 398 &, 715 \\
\hline C9 & 48,9091 & 47,338 &, 420 &, 712 \\
\hline C10 & 48,6060 & 46,276 &, 472 &, 705 \\
\hline C11 & 47,4059 & 49,724 &, 358 &, 719 \\
\hline C12 & 47,3004 & 50,189 &, 352 &, 720 \\
\hline C13 & 47,8327 & 49,038 &, 377 &, 717 \\
\hline C14 & 47,9182 & 49,033 &, 328 &, 722 \\
\hline C15 & 48,0735 & 47,960 &, 397 &, 714 \\
\hline
\end{tabular}

